\title{
Matemáticas y ordenadores en Arqueología. Una propuesta metodológica para trabajar con fragmentos cerámicos
}

\author{
Carmen Rísquez Cuenca*
}

En los últimos años los métodos cuantitativos han pasado a formar parte de la arqueología por el uso cada vez más frecuente de los mismos en los distintos estudios que conforman esta disciplina. Este trabajo no pretende ser en manera alguna un estudio exhaustivo de los mismos, ni mucho menos abordar de una manera amplia los conceptos estadísticos, ni la gran variedad de análisis existentes con aplicación a la arqueología, puesto que existen ya trabajos dedicados a ello DORAN y HODSON 1975, ALDENDEFER 1987, algunos traducidos ya al español como el de ORTON 1988 o el de SHENNAN 1992 entre otros, al mismo tiempo que en los últimos años han ido surgiendo revisiones, ensayos y aplicaciones por parte de investigadores españoles, CONTRERAS 1984, 1986, 1991, ESTÉVEZ y LULL 1984, FERNÁNDEZ 1985, BARCELÓ 1989 , LORRIO 199| etc... Pretendemos presentar un método de estudio para los fragmentos cerámicos a partir de técnicas cuantitativas, que hemos desarrollado en algunos trabajos RÍSQUEZ, HORNOS, RUIZ, MOLINOS 1991, RÍSQUEZ 1992, MOLINOS, RÍSQUEZ, SERRANO, MONTILLA 1995, pero no quisiéramos empezar sin un recorrido, tal vez algo rápido, de lo que han sido los métodos cuantitativos aplicados a la arqueología y su uso cada vez más extendido en nuestro país.

Si intentamos hacer una valoración histórica del uso de las técnicas estadísticas podemos ver cómo con anterioridad a la II Guerra Mundial, la preponderancia de la arqueología tradicional hacía inexistente la utilización de las matemáticas y con ello, de los principios de cuantificación. La aproximación teórica del arqueólogo se veía restringida a una "identificación de culturas espacio-temporales a partir de los vestigios materiales que la caracterizarán". Será tras finalizar la guerra, cuando empiecen a proliferar estas técnicas.

En Francia, ligados a la arqueología prehistórica de larga tradición en este país, empiezan a aparecer los primeros trabajos en los que se introducen técnicas gráficas y de estadística elemental. Muestra de ello son los trabajos de Bordes en los años 50, que utiliza diagramas acumulativos para identificar culturas prehistóricas, los de Spaulding en el año 1953, con los primeros cálculos de correlación, para evidenciar los tipos de objetos, o las primeras seriaciones para establecer cronologías relativas de Brainerd y Robinson en 1951, todos ellos expuestos en el trabajo de Djindjian 1984-85.

Si estos son los primeros intentos con estadísticas sencillas, será en la década de los 60, cuando los métodos cuantitativos en arqueología empezarán a adquirir importancia.

Ese camino hacia la cuantificación de los datos, está motivado por el deseo de eliminar la carga subjetiva del investigador y dotar a la Arqueología de un aparente carácter científico. Por ello, cuando Shenan 1992 habla de "Revolución cuantitativa", indica que ésta se llevó a cabo para dar un aspecto científico a la Arqueología y desligarla de su imagen tradicional.

*Área de Prehistoria. Universidad de Jaén 
Este desarrollo de la arqueología cuantitativa será el resultado de la convergencia de una serie de factores como:

I. El formidable impulso de las matemáticas aplicadas a los dominios científicos, gracias a la aparición de los ordenadores personales.

2. Desarrollo de un movimiento cuantitativo general en las ciencias sociales y ciencias de la naturaleza.

3. La aplicación de nuevas líneas en la arqueología, ya que veremos cómo se introduce en el campo de otras disciplinas, como Antropología, Geología, Física etc..

La aparición de los ordenadores personales va a ser de gran importancia ya que serán las herramientas que nos van a permitir llevar a acabo el análisis de la ingente cantidad de datos que se generan en el proceso de investigación y que nos van a ayudar a sintetizar toda esa información y a gestionarla de una forma más rápida, podríamos decir de una manera figurada que nos va a ayudar a jugar con nuestros datos. Shennan, apunta en su obra que "hay que reconocer que los arqueólogos empezaron a usar el ordenador como si de un jugete nuevo y excitante se tratara." Y que "la relación de la arqueología con los métodos cuantitativos procede de los ensayos realizados por parte de aquellos arqueólogos a los que les gustaron los nuevos juguetes y encontraron un uso para ello" SHENNAN 1992: p.19.

Otra serie de factores influirán también de una manera decisiva en su desarrollo. En E.E.U.U. surge por esos momentos la Nueva Arqueología que incorporará también la cuantificación a sus trabajos. Muchos arqueólogos están de acuerdo en que el trabajo de L.R. Binford y S.R. Binford del 1966, fue uno de los más importantes, al poner de manifiesto la importancia de aplicar los métodos cuantitativos en Arqueología, siendo de los primeros en utilizar los análisis multivariables. El trabajo que fue planteado para poder interpretar el carácter de las facies musterienses establecidas por F. Bordes, analizaba la variabilidad funcional a partir del estudio de una serie de niveles correspondientes a esa facies. Para ello, utilizaron un análisis factorial con el que demostraron la existencia de una serie de factores, cuya presencia se interpretaba por la funcionalidad específica de los útiles que lo formaban.

Siguiendo con las aplicaciones de análisis multivariables, una de las primeras aplicaciones del análisis cluster fue la de HODSON 1970, que realizó un trabajo sobre los niveles auriñacienses y perigordienses del SW francés junto con un análisis de componentes principales.

Sin embargo, esta década de los 60 es conocida principalmente por lo que supusieron los trabajos de CLARKE 1984, quien escribirá su obra más importante, Arqueología analítica, en 1968. Este trabajo, en el que se hace uso de conceptos procedentes de otras muchas disciplinas - geografía, estadística, geología, antropología etc..-, representó, en cuanto a metodología se refiere, el punto de inflexión definitivo de la arqueología europea, el comienzo de "su pérdida de la inocencia".

Este impulso que adquieren las técnicas cuantitativas, está unido, sin duda, a la difusión que durante estos años empiezan a tener los ordenadores. En su intento de objetivizar la información con la cuantificación de los datos, el arqueólogo llega a la observación de un gran número de variables sobre los artefactos en estudio buscando el medio de poder relacionarlas. Para ello, utiliza una serie de complejos algoritmos y largos cálculos matemáticos que limitaban, en cierto modo, su desarrollo. La incorporación del ordenador representará la solución de estos problemas, donde la estadística clásica deja paso a la estadística multidimensional y los modelos cualitativos reemplazarán a las viejas técnicas de aproximación.

Las matemáticas aplicadas "invaden", en palabras de DJINDJIAN 1984-85, todos los dominios científicos, y la Arqueología recogerá de éstas todos los aspectos que permitan su desarrollo hacia nuevos campos. Así, la Taxonomía numérica proveniente de la Ecología se aplicará a la Tipometría, dando como resultado el desarrollo de las Tipologías; las estadísticas multidimensionales análisis de 
datos, procedentes de la Psicometría permitirán las identificaciones culturales; el Análisis espacial proveniente de la Geografía conducirá a la Arqueología espacial, etc..

Durante estos primeros años se pone de manifiesto también, el difícil diálogo entre una Arqueología que acaba de salir de un mundo de textos y objetos, que utilizará los métodos matemáticos sin haberlos entendido plenamente, y una Arqueología matematizada que no acaba de situarse en la problemática arqueológica. Estos problemas, junto con el interés de un determinado grupo de arqueólogos por los métodos matemáticos, manifiestan la necesidad de una colaboración entre matemático y arqueólogo. La problemática se plasmará en la organización de reuniones, con objeto de iniciar los contactos entre aquellos investigadores que se decantan por las nuevas líneas, con el fin de presentar sus métodos de trabajo y establecer una puesta en común sobre el desarrollo de éstos métodos estadísticos en nuestra disciplina.

Los años 70 se inician, como señala CONTRERAS 1986: p. 27, con dos importantes obras referidas a este punto. La primera, Archaeologie et calculateurs de GARDIN en 1970, donde se recogen las comunicacciones del encuentro de arqueólogos que se celebró en Marsella un año antes, 1969, con dos fines:

- desarrollo de los métodos óptimos para el registro objetivo del material arqueológico, lo que requiriría el desarrollo de lenguajes analíticos - descriptivos.

- el uso del ordenador en los esfuerzos clasificatorios del arqueólogo, lo que hace necesario el desarrollo de los métodos estadísticos y de correlación aplicables a la naturaleza de la cultura, a las peculiaridades del material arqueológico y a las tareas de la investigación contemporánea.

La segunda recoge, asimismo, las comunicaciones de otra reunión, esta vez en Mamaia (Rumania), Mathematic in the archaeological and historical sciencices HODSON et al. 1970, en la que se tratará principalmente de los problemas de seriación y juicios matemáticos de similitud sobre la base de las matrices de datos.

Uno de los puntos más positivos que aporta esta arqueología cuantitativa es la toma de conciencia de la necesidad de formalizar los razonamientos en Arqueología. Ello irá vinculado al nacimiento de una arqueología teórica que sigue un desarrollo paralelo. Aparecen obras fundamentales como la de BINFORD 1972, "An Archaeological perspective", donde los artículos se dedican, sobre todo, a cuestiones metodológicas como la posición teórica de la que se parte para llegar a la interpretación de los materiales arqueólogicos, las técnicas que se pueden utilizar para la recogida de datos y que métodos se pueden seguir para la organización del material.

Hay que destacar también la periodicidad que adquiren los Advances in Archaeological method and theory, editados por Schiffer, desde 1978, donde aparecerán artículos referentes tanto a cuestiones teóricas como metodológicas, permitiéndonos seguir el continuo debate y el desarrollo de estas cuestiones.

Otras obras tendrán como tema central la aplicación de esos nuevos métodos cuantitativos a la Arqueología, DORAN y HODSON 1975, donde se apunta que, si bien la arqueología ha llegado a ser "científica", el arqueólogo debe primero poder describir con precisión y clasificar objetivamente los restos materiales que constituyen su principal dato. La necesidad de una descripción precisa justifica el énfasis sobre la cuantificación, y la necesidad de ser objetivo en su clasificación justifica el uso de las técnicas matemáticas. La recopilación de todos éstos métodos así como las posibilidades que ofrecen los ordenadores, constituyen el tema central de la obra.

De similares características, aunque de más fácil comprensión al no utilizar un lenguaje excesivamente técnico, es la obra de ORTON 1980, traducida al castellano en 1988, Matemáticas para arqueólogos. Ésta viene a argumentar por qué el discurso cuantitativo constituye un tema central para la 
Arqueología. Su finalidad es "demostrar que las ideas matemáticas se encuentran en el meollo de muchos conceptos e ideas arqueológicas, y exponerlas a través de ejemplos prácticos. No se pretende convertir al arqueólogo en un matemático o viceversa, pero sí mostrar qué tipo de trabajo se ha hecho y ayudar al lector para que consiga adentrarse en él" ORTON I988: I I- 12.

Para ello utiliza una serie de preguntas que constituyen la base del modelo de investigación que sigue un arqueólogo: ¿Qué es esto? ¿Qué antiguedad tiene? ¿De dónde procede? ¿Para qué sirve?, con las que demuestra cómo estos métodos cuantitativos nos pueden ayudar a alcanzar respuestas a una nueva pregunta ¿Hay un problema que resolver?, es decir, cómo interpretamos esos datos y que conclusiones sacamos.

Todos estos trabajos están poniendo de manifiesto la presencia del matemático en nuestra disciplina y su colaboración con el arqueólogo.

Una obra similar es Quantifying archaeology de SHENNAN 1988, traducida recientemente al castellano por Crítica, Arqueología Cuantitativa SHENNAN 1992, con un marcado carácter de manual, que se ocupa, en general, de la aproximación matemática y estadística a los datos arqueológicos através de numerosos ejemplos que conciernen a la cerámica de una forma mayoritaria; considera que la mejor manera de acercarse a aquellos que no son "técnicos" en matemáticas, es trabajando con ejemplos (se presentan ejercicios para su realización) mejor que introducirse directamente en la teoría, por ello el libro resulta ameno, algo difícil de encontrar en este tipo de manuales dedicados a aplicaciones estadísticas.

Durante todos estos años, la vinculación, cada vez mayor, de la cuantificación a la arqueología, queda patente no sólo en la gran cantidad de publicaciones que aparecen, sino también por el número de Reuniones y Coloquios que se llevan a cabo para el intercambio y exposición de trabajos, una puesta en común que manifiesta la gran rapidez con la que evolucionan las nuevas técnicas y el continuo desarrollo de la "inteligencia humana" buscando nuevos métodos que permitan un mayor acercamiento a los objetivos planteados: el estudio de las sociedades del pasado a partir de sus restos materiales de una forma objetiva.

Muestra de ello son los Coloquios británicos, con una periodicidad anual desde 1973, Computer Applications in Archaeology, que han pasado a denominarse últimamente Computer and Quantitative Methods in Archaeology; en E.E.U.U., Quantitative Research, ALDENDERFER 1987, el celebrado en Italia en 1988 Archeologia e Informatica, el Congreso mundial de Arqueología celebrado en Venezuela en 1990, Communication in Archaeology: a global view of the impact of information technology, REILLY y RAHTZ 1990, destacamos aquí el que se realizó en Madrid en 1990, Aplicaciones Informáticas en Arqueologia FERNÁNDEZ y FERNÁNDEZ |99|, o el European Symposium on Computing in Archaeology, celebrado en París en Noviembre 1991, o el Coloquio de Arqueometría que se celebrará próximamente en abril de este mismo año 1995 en Francia "L'archéométrie dans les pays européens de langue latine".

A éstos se unirían las revistas y publicaciones de carácter específico como Archaeo-logical Science, Science and Archaeology, Archaeometry ó American Antiquity.

\section{UNA VISIÓN DE LA ARQUEOLOGÍA CUANTITAVA EN ESPAÑA.}

FERNÁNDEZ 1989, ya hablaba del retraso con que las técnicas de cuantificación y en general la aplicación de Análisis estadísticos multivariables llegan a la Península. Para ello, no tenemos más que revisar la bibliografía que se publica sobre arqueología en nuestro país, para observar que, excepto algún estudio en los años 60, no es hasta finales de los años 70 principio de los 80 cuando hacen su aparición de forma más continuada, algunas técnicas de estadística simple aplicadas por parte de los paleolitistas que sufren la influencia de la 
escuela de Bordes y La Place, (MERINO 1980, BERNALDO de QUIRÓS 198I en CONTRERAS (986).

En las I Jornadas de Metodología de Investigación Prehistórica de Soria en 1981, auque las actas no se publicaran hasta 1984, se presentan un gran número de trabajos vinculados a la aplicación de las nuevas técnicas, casi todas ellas sobre conjuntos cerámicos, y con un predominio de la estadísticas simple. Sin embargo, estos trabajos reflejan ya un intento de cambio en el panorama de la Arqueología peninsular en un cierto sector de la investigación.

ESTÉVEZ y LULL 1984, expondrán un método basado en estadística sencilla, similar al que se utiliza en Biometría, basado en:

1. Aislar las variables (distintas dimensiones de las vasijas) que se consideren más definitorias.

2. Construir histogramas para cada variable y ver las posibles tendencias.

3. Calcular la media de la medida.

4. Calcular el coeficiente de variablidad obtenido a partir de la desviación "standard".

5. Establecer las relaciones entre variables, para lo que se pueden utilizar los índices y buscar, asimismo, los coeficientes de relación entre dos variables seleccionadas.

Con todo ello se realizarán los gráficos con los que se podría establecer la comparación entre medida y proporciones de Formas y Tipos distintos ESTÉVEZ y LULL 1984: pp. 237-238.

En la comunicación que presentan GRACIA junto a otros investigadores, GRACIA ET AL. 1984, se acercan al tema de la fragmentación cerámica, donde se tendrán en cuenta las características técnicas de la cerámica ( elaboración de la pasta, factura del vaso, cocción, acabado, técnica decorativa etc.), formales (bordes y fondos para los que se sigue la tipología de Dedet-Py, galvos, cuerpos, cuellos) y funcionales (suspensiones y asas), para establecer, con la aplicación de la técnica del Chi-cuadrado y calculando las distancias entre los diversos conjuntos, un dendrograma de los yacimientos estudiados.

Otro trabajo interesante, también presentado a estas Jornadas, es el de CHAPA 1984, donde se aprecia ya el interés por los análisis multivariables. Su estudio presenta un conjunto de fibulas de la Tène, con el que se pretende, a partir de las fijacción de tipos y la relación de éstos con las fases culturales, deducir cuál ha sido la evolución cultural de cada objeto y qué relación tienen con el resto. Para ello utilizará un dendrograma jerarquizado de las fibulas.

LULL 1983, utilizará el método ya presentado en Soria, aunque más detallado, en su trabajo sobre la Cultura del Argar, donde analizará el complejo cerámico de esta Cultura.

A este trabajo se le ha criticado especialmente, el criterio subjetivo seguido para separar las "formas", partiendo de las que habían sido establecidas con anterioridad por Siret, cuando el mismo autor había criticado los esquemas trazados por éste fuertemente. Es criticable, igualmente, el haber separado, a priori, el material doméstico del funerario, atribuyéndole, de partida, un criterio funcional sin tener en cuenta que también los materiales domésticos pueden formar parte del ritual funerario, por lo que hubiera resultado más objetivo tratar estadísticamente el conjunto completo, CONTRERAS 1986: 196

Anotemos que es también durante estos primeros años de la década de los 80 , cuando empiezan a aparecer un gran número de manuales sobre Análisis estadísticos, obras de carácter general que introducen al "inexperto arqueólogo" en el uso de estas técnicas CUADRAS 1981, SÁNCHEZ CARRIÓN 1984 etc..

Se inician en 1984 los Congresos de Teruel que son en cierto modo la continuación de las Jornadas celebradas en Soria, pero decantándose ahora por un tema: La Arqueología espacial. También aquí se van a presentar estudios en los que se aplicarán los 
métodos estadísticos, ahora a los asentamientos. Estos trabajos tendrán una mayor implantación en Teruel 87, donde se trató el tema del microespacio.

En el año 84 encontramos también los primeros trabajos de Contreras, en los que se tratará el tema de la Cuantificación en la Arqueología. En su primer trabajo CONTRERAS 1984, realiza una introducción al mundo de la clasificación y la tipología en arqueología, explicará el significado de la Taxonomía numérica y los modelos tipológicos en arqueología donde diferenciará los distintos enfoques en esta disciplina:

\section{Enfoque tradicional}

\section{Enfoque atributual}

3. Enfoque de agrupamiento de Ítems; donde se expondrán los distintos métodos multivariables y las principales técnicas de agrupamiento entre ellos.

Destacamos también los trabajos de ESQUIVEL y CONTRERAS 1984, donde se plantea la asociación del análisis Cluster con el análisis de Componentes Principales. Esta línea que abrirá el Dpto. de Prehistoria de la Universidad de Granada, aplicando los métodos cuantitativos a los registros materiales, se ve plasmada en el trabajo de CONTRERAS 1986 en el que aplicará los métodos cuantitativos, entre los que destacamos la asociación de un Análisis Cluster con un Análisis de Componentes Principales sobre el complejo cerámico de la Cuesta del Negro de Purullena, e iniciarán, igualmente, la aplicación de éstos análisis a otros campos como el de la analítica cerámica, más recientemente, CONTRERAS, CAPEL, ESQUIVEL, MOLINA, DE LA TORRE 1988.

Basándose en esta línea abierta por el Dpto. de Prehistoria de la Universidad de Granada, se enmarcarían los trabajos que se vienen desarrollando en las áreas de Prehistoria, Arqueología e Historia Medieval de la Universidad de Jaén, en la que desarrollamos nuestros trabajos, de los que hablare- mos al plantear la metodología para el estudio de fragmentos cerámicos.

Otros métodos, como el de la seriación automática y la combinación de varios análisis, han sido tratados por FERNÁNDEZ 1985 y BARCELÓ 1988. Resulta interesante el trabajo de éste último por tratarse de una contrastación de distintos análisis (de conglomerados, de regresión múltiple, de componentes principales y factorial) aplicados sobre un conjunto de estelas antropomorfas de la Península Ibérica. Después de la utilización de estos análisis, el autor se decanta por la heterogenidad del fenómeno de las estelas antropomorfas y las estatuas-menhir; para contrastarlas se decide, finalmente, por un análisis factorial donde, a través de los factores que se van a definir y su asociación, se obtendrán 4 clases, que vienen a demostrar la gran homogeneidad del conjunto de las estelas frente a la heterogeneidad de la Estatuas-menhir. El trabajo resulta interesante porque le confiere a la aplicación del (AF) una capacidad de resolución, a través de la diferenciación factorial en que se ponen de manifiesto las tendencias que se establecerán entre las variables que se introducen que permiten diferenciar los grupos.

La importancia que han adquirido los métodos cuantitativos aplicados a la Arqueología y el creciente interés por parte de los arqueólogos, se puso de manifiesto en lo que fue la primera reunión de ámbito estatal celebrada en Madrid en Octubre de 1990. En ésta se pudieron observar las líneas de investigación dentro del campo de la informática aplicada en la Arqueología, que se siguen en nuestro país.

Allí se puso de manifiesto el creciente interés del arqueólogo por la utilización de Bases de datos y Programas integrados. Esto está motivado por la implantación de los ordenadores personales de gran potencia, que permitirán el almacenamiento de gran cantidad de información. Se ha pasado, pues, de utilizar el ordenador de una forma puntual, a la posibilidad de una utilización diaria (archivo de datos en la excavación, inventarios, publicaciones..). La posibilidad de ordenación y de inves- 
tigación de los datos de los artefactos y de sus atributos que ofrece la Base de Datos, permitiendo la creación de ficheros especializados, es lo que ha movido al interés por estos métodos siendo uno de los que mayor repercusión ha tenido entre el colectivo de arqueólogos. Si bien ha tardado más que la Estadística en incorporarse, su éxito responde en gran medida a las posibilidades de poder gestionar rápidamente el conjunto global de las informaciones que poseemos sobre el registro arqueológico.

La línea que se sigue, es la de llegar a una integración de todos los datos de un proyecto arqueológico: materiales, topografía, estratigrafía, gráficos, fotografía, análisis, etc.. Esta línea es la que sigue ARCHEODATA ARROYO BISHOP 199I. En lo que atañe directamente a la Península, las bases de datos que más se utilizan son las de gestión e información de los datos cerámicos Fragmentos -

Dentro de los sistemas integrados, tendríamos que señalar el del Dpto. de Prehistoria de la Universidad de Granada, en el que teniendo en cuenta el tipo de información con el que suele trabajar el arqueólogo, articulará la información de textos, la gráfica y las imágenes gestionado por HYPERMAP - WINDOWS, con programas comerciales de bases de datos (DBIV y SUPERBASE4), hojas de calculo (LOTUS I, 2, 3 y EXCEL), datos que serán analizados con análisis multivariantes, textos (WORD PERFECT), al mismo tiempo que utilizan el tratamiento de los datos con el programa AUTOCAD para gráficos.

Otra de las líneas seguidas será la aplicación de análisis cuantitativos y la estadística, ya hemos indicado algunos de los trabajos desarrollados en esta línea a lo largo de los años 80. La mayoría de ellos se basan en paquetes de programas estadísticos comercializados que ofrecen al investigador la posibilidad de utilizar varios modelos de análisis. Aunque menos, también se realizan investigaciones originales, donde destacamos los trabajos de ESQUIVEL 1984, 1988, 1989, I991.
Poniéndose de manifiesto también la importancia que tiene la colaboración entre Matemático y arqueólogo, aunque desafortunadamente, no es algo muy extendido en nuestro país. Destacamos también los trabajos de FERNÁNDEZ Y FERNÁNDEZ I99।, que elaboran un programa para el tratamiento estadístico utilizando el DBASE IV, como punto de partida .

\section{EL ENFOQUE DE AGRUPAMIENTO DE ÍTEMS: LOS MÉTODOS MULTI- VARIABLES.}

El registro arqueológico puede proporcionar una gran cantidad de información que afecta a distintos campos, entre ellos la cultura material que se va a poner al descubierto, y que va a ser uno de los campos donde nosotros vamos a profundizar. Es precisamente esa "cantidad" lo que ha llevado al investigador a recurrir a la realización de clasificaciones y tipologías que faciliten su trabajo, y es ahí, donde se van a desarrollar y a aplicar los métodos de la taxonomía numérica.

En el camino de aproximación hacia la taxonomía, el arqueólogo ha adoptado distintas posturas, CONTRERAS 1984, 1994, diferencia, como hemos dicho anteriormente, dentro de los modelos tipológicos en arqueología y desde el punto de vista de la cuantificación y uso de métodos matemáticos, 3 enfoques distintos:

- El enfoque tradicional, caracterizado fundamentalmente por el carácter intuitivo de la elección de los TIPOS.

- El enfoque atributual, basado en el análisis de los atributos. Con él, se empezarán a deserrollar los métodos numéricos todavía de forma sencilla.

- El enfoque del Agrupamiento de Items, basado en los métodos matemáticos multivariables. El progreso que éstos van a alcanzar, permiten el desarrollo de este enfoque.

Nosotros hemos optado por este último, 
y vamos a tratar de justificar esta elección.

\section{EL POR QUÉ DEL ANÁLISIS MULTIVARIABLE.}

La investigación aplicada tanto a las ciencias naturales como a las ciencias sociales frecuentemente se encuentra con fenómenos complejos que van a requerir para ser explicados, el analizar una considerable cantidad de variables que han sido establecidas para definirlos. Por ello, interesará ver el comportamiento de todas esas variables, y cómo se interrelacionan, con lo que el tratamiento que se aplica a esos datos es multidimensional, recibiendo el nombre de técnicas, métodos o simplemente ANÁLISIS MULTIVARIABLE.

KENDALL 1975, define el análisis multivariable como el conjunto de técnicas que analizan simultáneamente más de dos variables en una muestra de observaciones. De una forma más amplia, se podría definir como "la rama de la estadística y del análisis de datos que estudia, interpreta y elabora el material estadístico sobre la base de un conjunto de $n>1$ variables que pueden ser de tipo cuantitativo, cualitativo o una mezcla de ambos" CUADRAS 198I.

Su objetivo fundamental es como ya hemos indicado en otros apartados, resumir y sintetizar grandes conjuntos de datos y variables, en función de ciertos objetivos, de cara a obtener informaciones válidas que permitan una mejor comprensión del fenómeno objeto de estudio.

El término MULTIVARIATE ANALYSIS, se ha traducido de formas distintas:

Análisis Multivariable: se ha utilizado generalmente en Psicología, Sociología y Economía

Análisis Multivariante: utilizado en Bioestadística, Biología, Economía

Análisis Multivariado: utilizado en Psicología y Educación

Si bien todas ellas, hacen referencia al mismo concepto. Nosotros utilizaremos el término "Multivariable", por entender que se aplica a más de dos Variables.
Para que este análisis tenga sentido hay que seguir un camino, determinado por tres fases:

I.- Un análisis exploratorio de los datos. Es decir, comprender cada una de las variables que vayamos a utilizar. Esto incluye, la estadística descriptiva univariable como medidas de tendencia central, variabilidad, representaciones gráficas, etc..

2.- Buscar la relación entre pares de variables. Para ello, la técnica más utilizada es la Correlación de Pearson.

3.- Una vez conocemos las variables que vamos a utilizar, las analizaremos simultáneamente, pudiendo trabajar con una parte o con todas ellas, según los objetivos que nos marquemos.

Respecto al tipo de método que resulta más conveniente usar una vez realizado un estudio previo de las variables que se van a utilizar, dependerá del investigador. Diversos autores han propuesto una clasificación de los métodos multivariables, sin embargo, la labor resulta difícil dada la diversidad y los distintos enfoques clasificatorios que éstos presentan. Una primera distribución podría establecerse en base a:

\section{MÉTODOS DESCRIPTIVOS O EXPLORATORIOS.}

Con éstos, el investigador se aproxima a los datos sin ninguna hipótesis previa. Se observará la realidad explorando los datos en busca de nuevos conocimientos. Aquí se incluirán el Análisis Factorial, el Análisis Cluster, el Análisis de Correspondencias y las Escalas Multidimensionales.

\section{MÉTODOS EXPLICATIVOS O CONFIRMATORIOS.}

A diferencia de los anteriores se basan en un marco teórico que fundamenta y justifica una hipótesis previa que intentan validar empíricamente. Formando parte de éstos estarían, el Análisis Multivariable de Varianza, Regresión Múltiple, Análisis Causal, Análisis Discriminante. 
En la Fig. I. recogemos el intento de clasificación de Evrard y La Maire, realizado a partir de análisis anteriores de otros autores, donde el principal criterio clasificatorio es el objetivo del investigador.

Recogiendo una serie de aportaciones en este sentido, BISQUERRA 1989, propone una clasificación de los métodos multivariables, Fig. 2 donde los criterios clasificatorios son:

- Métodos descriptivos o explicativos

- $N^{\circ}$ de variables dependientes

- Escalas de medida

Al mismo tiempo presenta otra clasificación, en la que se toma como primer criterio clasificador, el propósito del análisis. El resultado de ésta, lo presentamos en la Fig. 3, donde se pueden observar los distintos métodos que podemos utilizar dependiendo de nuestro objetivo, (contrastar, correlacionar, predecir, describir, ó ajustar modelos).

Con ello podemos tener una visión general sobre los distintos análisis multivariables que podemos utilizar y el propósito por el que seleccionamos uno u otro en función del objetivo del trabajo que vayamos a realizar.

De todos estos análisis hemos seleccionado tres para aplicarlos sobre un conjunto de fragmentos cerámicos con el fin de elaborar una tipología en base a criterios morfométricos:

- Análisis Cluster, que nos permita fijar los tipos, clasificarlos y describirlos.

- Análisis Factorial, que nos permitirá a través de la reducción de la cantidad de información que manejamos, fijar las distintas tendencias que presente la muestra.

- Análisis Discriminante, que nos permita contrastar y confirmar los resultados establecidos, al mismo tiempo que predecir en trabajos futuros la clasificación de nuevos elementos.

\section{EL ANÁLISIS CLUSTER: LA FIJACIÓN DE TIPOS.}

El nombre de Análisis Cluster, se ha utilizado para definir una serie de técnicas, fundamentalmente algoritmos, que tienen por objeto la busqueda de grupos similares de individuos o variables que se van agrupando en conglomerados. Dada una muestra de elementos, sobre los que hemos realizado individualmente una serie de observaciones, el "ACL", nos servirá para clasificarlos en grupos, lo más homogéneos posible en base a las variables que hayamos observado, donde los elementos que se hayan quedado clasificados en el mismo grupo, seran tan similares como sea posible.

Al iniciar este análisis deben haberse tomado tres decisiones:

I.- Seleccionar las variables relevantes para identificar los Grupos.

2.- Elegir la medida de proximidad entre individuos, (básicamente similitudes y distancias).

3.- Elegir el criterio para agrupar a los elementos en conglomerados. Se trata aquí de seleccionar qué técnica de agrupamiento se considera más adecuada por el investigador para aplicar a su estudio.

Dentro del Análisis Cluster hay varios procedimientos, la mayoría empiezan a partir de una matriz de similitudes o distancias que consiste en una tabla de doble entrada donde aparecen los artefactos en las filas y en las columnas representándose en su interior los valores o índices de similitud entre cada par de variables. El coeficiente de un objeto consigo mismo es siempre 0 , lo que indica el mayor índice de similitud, y el coeficiente de similitud entre un artefacto " $x$ " y otro " $y$ " es el mismo que entre "y" e " $x$ " lo que nos indica que la matriz es simétrica, por lo que se prescinde normalmente de media matriz.

Según el concepto de similitud o distancia que empleemos nos resultará uno u otro dendrograma. Nosotros hemos utilizado uno 
de los más usados por los arqueólogos, el ANÁLISIS CLUSTER DE PROMEDIO NO PONDERADO (AVERAGE LINKAGE). En este, durante el enlace o encadenamiento permite a una unidad juntarse a un Cluster únicamente si su promedio de similitud con todos los miembros existentes alcanza un nivel específico, aquí la distancia entre dos grupos viene dada por la existente entre dos puntos calculados internos al grupo, CONTRERAS 1984: p. 357.

En la Fig. 4, presentamos un esquema de los principales métodos de Cluster Análisis, según BISQUERRA 1989:4I0.

\section{EL ANÁLISIS FACTORIAL: LA FIJACIÓN DE TENDENCIAS}

Esta técnica, consiste en resumir la información contenida en una matriz de datos con $\checkmark$ variables. El objetivo que se persigue, consiste en identificar un número de factores, inferior al número de variables, mediante los cuales se pueda describir el fenómeno observado de forma simplificada, es decir, reducir la información recogida. Para que el Análisis Factorial tenga sentido, deben cumplirse dos condiciones básicas:

I.- Los fenómenos deben explicarse con el menor número de elementos posibles. Por tanto, el número de factores debe ser lo más reducido posible.

2.- Estos factores deben ser susceptibles de interpretación sustantiva.

Ya hemos señalado con anterioridad, que trabajar con un número considerable de elementos y de variables, es sumamente laborioso, aunque con la llegada de los ordenadores, estos cálculos han dejado de tener tanta importancia. Esto es lo qua ha contribuido a que este tipo de análisis se haya convertido en una de las técnicas más usadas en la investigación de las ciencias sociales, y también en el campo que nos ocupa.
En el Análisis Factorial, se siguen una serie de pasos que podemos sintetizar de la siguiente forma:

- Calcular la matriz de correlaciones entre todas las variables a partir de datos originales, procediendo al examen de esa matriz.

- Extraer los factores que se consideren necesarios para representar los datos.

- Rotar los factores con objeto de facilitar su interpretación.

- Calcular las puntuaciones factoriales para cada individuo.

El Análisis Factorial intenta separar dos aspectos en los datos:

I. los efectos específicos

2. los efectos comunes

Cada factor se considera como un eje en el espacio al que se refieren los atributos. La orientación de esos ejes de referencia es arbitraria y se requieren una reglas que los fijen de una manera conveniente. Es conveniente señalar aquí, la importancia que tiene la rotación factorial, puesto que ésta pretende seleccionar la solución más sencilla e interpretable. Consiste en hacer girar los ejes de coordenadas que representan a los factores hasta conseguir que se aproximen al máximo a las variables en que están saturados. Con ello, la rotación de factores en el espacio transforma la matriz factorial inicial en otra, la Matriz factorial rotada, que ofrece una interpretación más fácil. En nuestro proceso de interpretación, deberemos estudiar la composición de las saturaciones factoriales más significativas para cada factor y de acuerdo con ellas, intentar dar un nombre a cada uno de los factores.

\section{EL ANÁLISIS DISCRIMINANTE: CONFIR- MACIÓN Y CONTRASTACIÓN.}

Podemos definirlo como una técnica de clasificación y asignación de un individuo a 
un grupo conocidas sus características. En este análisis, se dispone de una serie de grupos (Tipos en nuestro caso), definidos "a priori", con una serie de observaciones para cada individuo referidas a un conjunto de variables relevantes (en nuestro caso, los tipos habrán sido previamente definidos por el $\mathrm{ACL}$ ). En base a esta información se llega a calcular una función discriminante que se puede utilizar para hacer predicciones futuras. Se pueden comparar un grupo y otro con objeto de discernir cuales son los factores que mejor contribuyen a explicarlos.

Su objetivo podríamos resumirlo en:

- Determinar si en función de las variables originales disponibles, los grupos quedan suficientemente discriminados, explicándose así la diferenciación entre los grupos.

- Analizar cuáles son las variables que contribuyen más a discriminar entre los grupos que se han formado. Para ello, "se reducen" las variables que mejor discriminan a unas pocas nuevas variables que se denominan "Variables Canónicas", generalmente una única variable canónica es la que aporta mayor explicación. Las variables canónicas son combinación lineal de las variables originarias y vienen expresadas por una "función discriminante".

Cuando disponemos de un número considerable de variables, pero no sabemos cuáles de ellas contribuyen mejor a discriminar entre los grupos, se utiliza el método "STEPWISE" (que es el que hemos utilizado con el Paquete de programas BMDP, con el que se han realizado los análisis), éste selecciona a las variables paso a paso.

Este análisis nos va a permitir en nuestro caso, confirmar y contrastar los resultados que habíamos obtenido en los análsis anteriores, $A C L$ y $A F$, al mismo tiempo que nos permite modificar si lo consideramos conveniente, la clasificación para establecer la tipología final.

\section{LA APLICACIÓN DEL MÉTODO: DE FRAGMENTOS A TIPOS}

No cabe duda que el material más abundante en una excavación, es la cerámica ya que de todos los artefactos que nos pueden aparecer, ésta es la que aparece en mayor cantidad en el registro arqueológico, y cuando nos planteamos trabajar sobre esos materiales cerámicos, lo más frecuente es el estado fragmentario en que aparecen esos restos. De hecho, a no ser que se haya producido un abandono súbito, con buenas condiciones de conservación a lo largo del tiempo (no se hayan producido ocupaciones sucesivas en el lugar, la labor antrópica no haya ocasionado grandes daños etc.) o se trate de conjuntos cerrados, como pueden ser las necrópolis, lo normal es encontrar una gran cantidad de fragmentos cerámicos.

De partida, el número de éstos en sí, ya "asusta" al investigador a la hora de enfrentarse al estudio de tales restos (pongamos por ejemplo la cantidad de fragmentos cerámicos que componen el registro maestro del yacimiento prehistórico de El Malagón, 56.540. CONTRERAS, MOLINA, ESQUIVEL 1991. Si a esto unimos la imagen de información fragmentaria y desigual que éstos transmiten, hace que los fragmentos cerámicos se vean relegados a un segundo plano (listado de inventarios, anexos de catalogación) frente a los recipientes completos o en su caso aquéllos en que es reconocible la mayor parte de la forma, sobre los que se realizan los intentos tipológicos.

Una pregunta que se han planteado muchos investigadores es: ¿Podemos decir algo sobre la forma de un recipiente a partir de un fragmento?. Esta pregunta está formulada de manera simplista, y debemos ir en su formulación más allá, pues un fragmento en sí descontextualizado no nos dice nada. Los fragmentos aparecen en un espacio (yacimiento -ya sea en superficie o estratificado-, estructuras habitacionales, o áreas de actividad según la escala en que estemos trabajando). Están pues dotados de una capacidad para informar sobre un proceso socioeconómico. La labor del investi- 
gador, es convertirlos de simples objetos cerámicos en productos historizados. La pregunta pues sería ipodemos a partir de los fragmentos cerámicos decir algo sobre el contexto en que aparecen, que nos ayude a la reconstrucción del proceso histórico en el que éstos se han visto inmersos?.

Hemos tratado de dar respuesta a esta pregunta' trabajando con los fragmentos cerámicos a varios niveles:

- Macro espacio: El territorio

- Semi-micro: El Yacimiento

- Micro espacio: Las casas

Y para ello hemos definido una serie de variables morfométricas que expondremos a continuación junto con los métodos matemáticos multivariables que se aplicarán sobre ellas. De este modo, un conjunto de artefactos que se ven unidos por las relaciones que muestran sus variables métricas en la comunalidad de sus medidas y la interrelación que se produce entre las mismas, formarían el TIPO. La estructura de estos Tipos, es decir las Variables que los definen, constituyen el GRUPO TIPOLÓGICO. Por debajo del nivel TIPO, las variaciones que se produzcan dentro de ellos, es lo que denominaremos SUBTIPOS y VARIANTES.

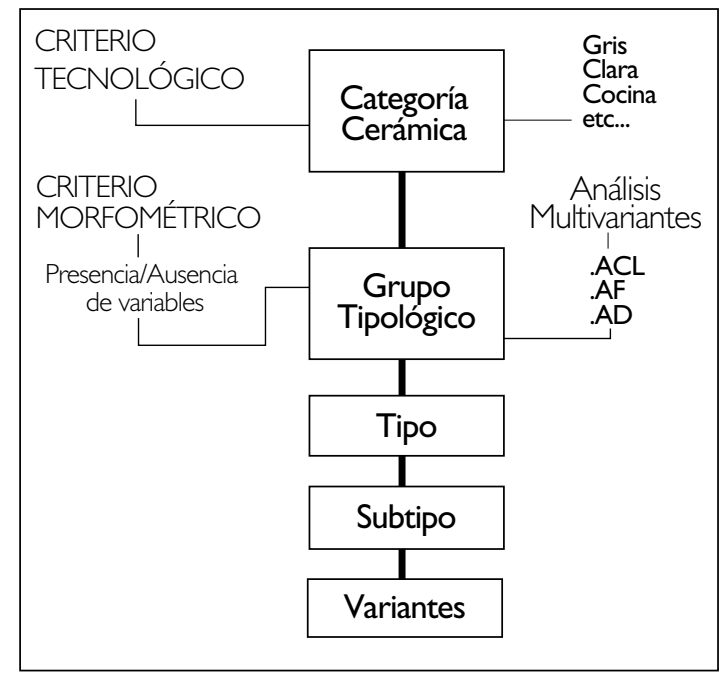

Fig. 5. Matriz tipológica
Sobre esta matriz que intenta la articulación de dos aspectos, el técnico funcional y el morfométrico, es en la que ha venido trabajando el Area de Prehistoria de la Universidad de Jaén, bajo la dirección del Dr. A. Ruiz. Fruto de ello, han siso varias Memorias de Licenciatura CHOCLÁN 1984, HORNOS 1984, LÓPEZ ROZAS 1984, GUTIÉRREZ 1994 y Tesis Doctorales MOLINOS 1987, NOCETE 1989, RÍsQUEZ 1992 y otros trabajos MOLINOS, RÍSQUEZ, SERRANO, MONTILLA 1995.

Al mismo tiempo, entre los fragmentos con forma, hemos seleccionado los bordes, a los que hemos unido las bases, por entender que ambos nos pueden proporcionar una mayor cantidad de información a la hora de intentar acercarnos a las FORMAS y con ello a la FUNCIONALIDAD DE LOS RECIPIENTES. El no haber elegido las asas, o elementos de sujeción, se debe a que aparecen en un número muy reducido en el registro cerámico que aquí estudiamos, por lo que no resulta útil aplicarle métodos multivariables.

LOS BORDES. Para llegar a una definición lo más exacta posible de esta parte de cualquier recipiente cerámico, a través de la morfometría, hemos fijado cuatro bloques de variables, que son definidas a partir de una serie de puntos localizados sobre el fragmento (Fig. 6). Los tres primeros bloques nos van a permitir trabajar sobre el total de la muestra a partir de una serie de puntos que definimos de la forma siguiente:

A. Punto de contacto del borde con el plano horizontal.

B. Punto más exterior del borde.

C. Punto obtenido al proyectarse "A" perpendicular al plano de la boca sobre la pared interior o exterior del fragmento.

D. Punto obtenido al proyectarse "B" horizontal al plano de la boca hacia la pared opuesta del fragmento.

\footnotetext{
I De hecho se han realizado varias Tesis, MOLINOS 1987, NOCETE 1990, RÍSQUEZ 1993, Memorias de Licenciatura, CHOCLÁN 1984, HORNOS 1984, LOPEZ ROZAS, 1984, GUTIERREZ 1994, y Trabajos, MOLINOS; RÍSQUEZ; SERRANO; MONTILLA I995, desarrollando el método.
} 


\section{E. Punto de gravedad.}

\section{El Primer bloque. Las variables: $\mathrm{X}, \mathrm{X}^{\prime}$ e $\mathrm{Y}$, verticalidad y horizontalidad.}

Un primer bloque de variables pensado para valorar la horizontalidad y/o verticalidad de los bordes, al mismo tiempo que el tamaño de los mismos es el compuesto por $X$ e $Y$. La primera de ellas se define como la medida de la proyección A-C. Esta primera medida pone de manifiesto dos tipos diferentes de bordes, ya que en su proyección perpendicular desde el plano horizontal hasta la pared del fragmento, el punto $C$ puede tocar la pared exterior del mismo, en cuyo caso denominaremos a la variable como $X$, o puede tocar en la pared interior $y$ en ese caso la denominaremos $X^{\prime}$. Ambas variables, son excluyentes, puesto que nos están definiendo dos formas distintas, la primera nos muestra los bordes de carácter abierto y la segunda los de carácter cerrado, lo cual no implica que el recipiente pueda ser cerrado o abierto.

Esta variable, nos va a permitir valorar la verticalidad y el tamaño del borde.

La segunda variable, la Y, nos permite valorar la horizontalidad y con ello el grosor del borde. Ésta se define en aquellos fragmentos con variable $X$, como la medida de la proyección de $\mathrm{B}-\mathrm{D}$, es decir, partiendo del punto más exterior del borde situado siempre a partir de la perpendicular tangente al plano más exterior (Punto B) se proyecta paralelamente al plano de la boca alcanzando en un punto la pared más interior (Punto D). Si el fragmento presenta $X^{\prime}$, la variable, partiría del punto más interior y alcanzaría en su proyección la pared exterior.

\section{El segundo bloque de variables: El punto de gravedad.}

Lo que definimos como bloque nos sirve aquí, para encontrar el punto de gravedad del borde, o sea, el punto de encuentro de las variables $X-Y \circ X^{\prime}-Y$. El resultado son cuatro nuevas variables que se definen a partir de su cruce, la variable vertical se ha divi- dido ahora en W y W'; la primera desde el punto $A$ al punto de encuentro, $y$ la segunda desde éste al punto $C$. La horizontal se divide a su vez en dos $V y V^{\prime}$, en función del mismo proceso. Las cuatro variables son reiterativas en su acumulación por parejas de las dos variables anteriores $(X$ e $Y$ ), de ahí su presencia o no en los análisis realizados ya que en los trabajos que hemos venido realizando para llegar a la definición de tipologías hemos comprobado si nos pueden ser o no de utilidad.

\section{El tercer bloque: la trayectoria final.}

Éste fue definido pretendiendo valorar el engrosamiento hacia el interior o exterior 0 en todo caso la forma extrema del borde, al mismo tiempo que refleja el tamaño. Para ello se fijaron cuatro variables más; la variable I/2 de $A B$, abarca como se indica la mitad de la distancia entre ambos puntos, repitiéndose el proceso para I/2 de AD. La proyección en ángulo recto de estas variables hasta topar con las paredes exterior e interior respectivamente, se ha definido como R para la primera y $S$ para la segunda.

\section{El cuarto bloque: Concavidades y convexidades.}

Este último bloque trata de medir las concavidades interiores y exteriores de los sectores del recipiente que contienen el borde, para ello trabajamos sobre seis variables más, cuya presencia o ausencia, junto con las anteriores variables definidas nos permitirán fijar los distintos grupos sobre los que vamos a trabajar.

Las variables $\mathrm{M}$ y $\mathrm{N}$ : Las concavidades. Éstas, nos van a permitir valorar la concavidad interior. Su obtención sigue un complejo proceso debido al tamaño del fragmento y a la verticalidad del mismo. La M, se obtiene trazando desde el punto A una tangente al punto más interior de la pared interior (PUNTO G), desde ella se proyecta una paralela que pase por el punto más exterior de la pared interior (PUNTO E). La variable se define desde éste hasta el punto en que el trazado de la paralela muestra mayor concavidad. 
Su proyección en ángulo recto hasta topar con la pared interior da lugar a la variable $\mathrm{N}$. Define generalmente a los recipientes de carácter abierto.

Las variables $P$ y Q: El exvasamiento. Para aquellos recipientes que muestran exvasamiento del borde,la medición de la concavidad se expresa en las variables $\mathrm{P}$ y $\mathrm{Q}$ ahora respecto a la pared exterior. Como en el caso anterior la tangente se proyecta desde el Punto $A$ al punto más interior de la pared exterior (PUNTO I), y la paralela a éste se hace pasar por el punto más exterior (PUNTO H), que no necesariamente coincide con el PUNTO B, por el giro propuesto por la tangente. En el punto de máxima concavidad y desde $\mathrm{H}$ se localiza la variable $P$ y desde ahí hasta topar con la pared exterior la $\mathrm{Q}$.

Las variables J y K: Recipientes cerrados con labio entrante. Las encontramos en aquellos recipientes de estas características, con presencia de $X^{\prime}$, cuando al trazar una perpendicular al punto más exterior del labio, (entendido éste como el tramo final del borde), se genera una concavidad medible en la pared exterior del borde, se toma en el punto central, de tal modo que, desde ese punto hasta el contacto con la perpendicular la denominaremos $\mathrm{K}$ y desde ese punto de contacto hasta el más exterior señalado la llamaremos J.

En función de los expuesto hasta ahora, la presencia o ausencia de estas variables nos determina la división de la muestra en 5 Grupos Tipológicos, siendo las variables que nos van a permitir su diferenciación las siguientes:

$$
X-X^{\prime} ; P-Q ; M-N ; J-K
$$

De donde obtendríamos los siguientes Grupos Tipológicos (Fig. 7):

GRUPO TIPOLÓGICO I.- Presencia de X, P y Q: Recipientes de carácter generalmente cerrado con borde exvasado.
GRUPO TIPOLÓGICO II.- Presencia de X, $M$ y $\mathrm{N}$ : Recipientes de carácter abierto de borde continuo, tendencia a exvasarse o ligeramente entrante.

GRUPO TIPOLÓGICO III.- Presencia de X, $\mathrm{P}, \mathrm{Q}, \mathrm{M}$ y $\mathrm{N}$ : por lo general recipientes de carácter abierto con bordes muy exvasados, tendiendo a la horizontalización.

GRUPO TIPOLÓGICO IV.- Presencia de X', J y K: Recipientes cerrados de borde entrante.

GRUPO TIPOLÓGICO V.- Presencia de X', sin ninguna de las variables del cuarto bloque.

LAS BASES. Al igual que para los fragmentos de bordes, también para los fragmentos de bases, hemos elaborado una serie de variables morfométricas que nos permitan en su conjunto una descripción formal lo más aproximada posible de esta parte del recipiente.

En todo fragmento de estas características, hemos de destacar una serie de puntos (Fig. 8):

I.- La superficie de contacto con el plano horizontal de base. Esta superficie puede estar conformada por un solo punto de contacto o por múltiples (un plano). A esta superficie la llamaremos a-a', siendo a el punto más exterior de la base en contacto con el PB (Plano de base), y a' el punto más interior de ésta, en contacto con el PB. En aquellas que sólo tengan un punto de contacto, la distancia a-a' será 0 , considerándose como un único punto.

2.- Un segundo punto a destacar sería aquél en que empieza la superficie, o el Plano interior de lo que consideramos Fondo de un recipiente, a este punto lo denominaremos $\mathbf{d}$, y es el resultado de la proyección perpendicular de a hacia la pared interior del fragmento.

3.- Un tercer punto, desde donde empezamos a denominar la parte del recipiente como base, considerado pues como el sector III-IV (RUIZ et alii, 1986) sería el resultado de 
proyectar el punto anterior $\mathbf{d}$ sobre la pared exterior del fragmento, por lo que denominaremos al nuevo punto d'.

Una vez definidos estos puntos, y a partir de ellos, hemos trabajado con un conjunto de siete variables cuyo proceso pasamos a definir a continuación (Fig. 8).

\section{VARIABLE I. DISTANCIA a-d.}

La proyección del punto a hasta tocar con la pared interior del fragmento al que hemos denominado $\mathbf{d}$ nos ofrece una distancia, cuyo valor da como resultado la altura del sector.

\section{VARIABLE 2.- DISTANCIA d-d'.}

Es la proyección desde el punto d hacia la pared exterior, donde marcamos el d'. El resultado es un plano imaginario al que consideramos inicio de la base como sector.

Para valorar las tres variables siguientes, partimos de una distancia que trazamos desde el punto d' al a, que nos permitirá valorar en principio dos grupos diferentes:

- Un grupo en que la línea pasará al exterior de la pared externa.

- Otro grupo en que ésta será interior a esta pared.

En el primero de ellos y teniendo en cuenta las dos primeras variables establecidas, se nos define un nuevo punto: el que nos marca la máxima concavidad en la pared exterior de la base, o punto de inflexión a partir del cual empezaría el pie como tal parte dentro de ésta. Este punto puede ser más INTERIOR al punto a, y en ese caso lo denominaremos c, o puede quedar al EXTE$\mathrm{RIOR}$ de a y en ese caso lo llamaremos e. El que esté presente uno u otro punto, genera dos grupos distintos.

\section{VARIABLE 3. DISTANCIA c-c' o e-e':}

Nos marcan la máxima concavidad de la base, es decir la inflexión que se produce al marcarse el pie del recipiente. La obtención de esta variable es el resultado de proyectar en ángulo recto desde el punto más interior de la pared exterior (ya sea c $\circ$ e), hasta tocar con la línea trazada. Al punto resultante le llamaremos c' o e'.

\section{VARIABLE 4. DISTANCIA d'-c' ó d'-e'.}

Es la distancia que hay desde el inicio de la base como sector hasta la proyección del punto de inflexión donde hemos marcado la máxima concavidad y empieza a definirse el pie como tal.

Esa línea que hemos marcado, nos genera además un nuevo punto al chocar en su trayectoria hacia a con el más exterior de la base localizado en el pie de éste. A este punto lo llamaremos b.

\section{VARIABLE 5. DISTANCIA b-b'.}

Desde el punto exterior del pie (es el más exterior al punto a), proyectamos hasta chocar con la distancia ya definida a-d.

La convexidad, aparecía cuando obteníamos una línea interior, ésta, nos permitirá valorar la máxima convexidad, donde puede o no tomar forma de pie, lo que estará en función de la presencia o no de una distancia a-a', esto nos permitirá la diferenciación de dos nuevos grupos tipológicos, bases planas o en su caso, con pie poco indicado y generalmente onfaladas.

\section{VARIABLE 3. DISTANCIAS f-f', $g-g^{\prime}$.}

Nos permite valorar la máxima convexidad de la base. Es el resultado de medir la distancia desde el punto más interior de la pared exterior hasta tocar con la línea d'-a.

\section{VARIABLE 4. DISTANCIAS f'-d', g'-d'.}

Es la distancia desde el inicio de la base como sector hasta el punto que, proyectado, marca la máxima convexidad.

En estos grupos está ausente la variable bb', pues son en general bases sin pie o con un escaso desarrollo de éste, por lo que el punto f, sería el que nos está marcando la función del pie como tal parte de la base.

Las bases planas, también pueden ser cóncavas, esto da lugar a dos nuevas variables $\mathrm{h}$ - 
h' y h'-d', que conformarían la presencia de un nuevo grupo tipológico.

Las dos últimas variables que tomaremos, también al igual que las dos primeras son aplicables a aquellos grupos que presentan concavidades medibles al interior, generadas por el pie o el rehundimiento de la base.

\section{VARIABLE 6. 0.5 DE a.}

\section{VARIABLE 7. I DE a.}

Tomando como referencia el punto a, trazaremos al medio centímetro $(0.5 \mathrm{cms}$. $)$ y a uno (I cms.) de éste, su proyección hacia la parte interior de la pared exterior de la base, con ello pretendemos valorar la mayor o menor altura y forma que genera al interior el pie en función de la trayectoria de sigue esa parte de la pared de la base.

Con todo ello, hemos obtenido 5 Grupos Tipológicos (Fig. 9) determinados por la presencia o ausencia de estas variables. Éstos, los podemos resumir en:

GRUPO TIPOLÓGICO I. a-d. d-d'. d'-c'. cc'. b-b'. 0.5a. Ia.

GRUPO TIPOLÓGICO II. a-d. d-d'. d'-e'. ee'. b-b'. 0.5a. Ia.

GRUPO TIPOLÓGICO III. a-d. d-d'. d'-f'. f-f'. 0.5a. Ia.

GRUPO TIPOLÓGICO IV. a-d. d-d'. d'-g'. g-g'.

GRUPO TIPOLÓGICO V. a-d. d-d'. d'-h'. h-h'.

Para realizar todos estos análisis, se ha utilizado el Paquete de Programas BMDP STA-
TISTICAL SOFWARE. Revisión 2.22, de 1983, de donde se han seleccionado El Cluster Análisis de Casos (2M), El Análisis Factorial (4M) y el Análisis Discriminante (7M).

Los programas han sido corridos, en un Ordenador DATA GENERAL ACLIPSE MV / 4000, con Sistema Operativo AOS / VS Rev. 7.65, del Servicio de Informática de la Universidad de Jaén.

\section{LOS TIPOS EN EL TIEMPO Y EN EL ESPACIO}

Como se ha indicado, hemos tenido la oportunidad de desarrollar la metodología aquí propuesta para el estudio de fragmentos cerámicos en una serie de trabajos ${ }^{2}$ donde se ha podido valorar su aplicación a varios niveles: Macroespacial: el Territorio, Semimicro: el Yacimiento, y Microespacial: las casas. Lo que exponemos a continuación, sin extendernos demasiado ${ }^{3}$, son algunos de los resultados obtenidos.

\section{EL TERRITORIO}

Se trabajó sobre un conjunto de cerámicas reductoras (grises y cocina) de un total de 12 yacimientos, pertenecientes la mayoría de ellos al Proyecto de Investigación "El Poblamiento ibérico en las Campiñas del Alto Guadalquivir" dirigido por los Dres. A. Ruiz y M. Molinos, contando con las estratigrafías obtenidas durante el proceso de excavación de los mismos, y seleccionando a la vez yacimientos de otras áreas vinculadas culturalmente aunque no en el mismo ámbito territorial con el fin de contrastar la información y observar su comportamiento, teniendo en cuenta que se valoraban yacimientos con funciones distintas: Oppida, Torres, Yacimien-tos en Ilano, Necrópolis, y Santuarios, representativos de

\footnotetext{
2 RÍSQUEZ, HORNOS, RUIZ, y MOLINOS I99I; RÍSQUEZ 1993; MOLINOS, RÍSQUEZ, SERRANO y MONTILLA I995, todos ellos, hay que decirlo, son materiales correspondientes a la cultura ibérica, en un amplio marco cronológico desde fines s. VII a.C al s. I a.C.

3 Estos trabajos se encuentran ampliamente desarrollados en la bibliografía comentada en la nota 2.

4 RISQUEZ, C. (1993): Las cerámicas de cocción reductora en el Alto Guadalquivir durante la época ibérica: Hacia una tipología contextual. Tesis Doctoral. Universidad de Granada. Microfichas.
} 
los modelos de asentamiento que podemos encontrarnos dentro de esta etapa cultural.

El trabajo se realizó partiendo por separado de las cerámicas grises y de las de cocina, separando bordes de bases, e incluyéndolos en los Grupos Tipológicos correspondientes según la presencia o ausencia de las variables establecidas. Las figuras que se presentan a continuación (Fig. I0,II) son un ejemplo de los resultados obtenidos para algunos de los grupos tipológicos. Las conclusiones a las que llegamos nos permitían establecer diferenciaciones de los grupos formales resultantes en el tiempo y en el espacio. Uno de los primeros resultados corroboraban la existencia de la frontera en el s. VI a.C. que separaría el mundo de la Campiña de Jaén del horizonte Tartésico, (frontera que ya se habia definido en los estudios de territorio ${ }^{5}$ ). Presentamos aquí dos ejemplos, el Grupo Tipológico I de las cerámicas de cocina (Fig. I0) donde los yacimientos con elementos de este periodo al que acabamos de referirnos, presentan a nivel de subtipos diferencias marcadas y se presentan separados en el análisis factorial según pertenezcan al ámbito de Tartessos (Llanete de los Moros, Las Calañas de Marmolejo) Subtipo 1.8 o al ámbito de La Campiña (Atalayuelas) Subtipo 1.6. Al mismo tiempo que podíamos observar la evolución de determinadas formas en el tiempo, como se puede apreciar en el caso de las bases de cerámicas grises del Grupo Tipológico II, donde observamos un aumento en la altura del pie en el transcurso del tiempo, al mismo tiempo que se marcan claras diferencias en los yacimientos (ausencia de algunos en los que estaban representadas únicamente las fases más antiguas y que carecen de este Gupo Tipológico, o su presencia mayoritaria en el Santuario ibérico de Castellar de Santisteban, donde podríamos atribuirlo a un carácter "funcional" relacionado quizás con las ofrendas (Fig. II), o la presencia en algunos yacimientos de tipos exclusivos.

\section{EL YACIMIENTO.}

En este caso se trabajó sobre el yacimiento de Las Calañas de Marmolejo ${ }^{6}$, un pequeño asentamiento ibérico en llano, con una cronología entre el 650 - 575 a.C.

Se analizaron un total de 244 fragmentos, entre bordes y bases, seleccionados de los cortes estratigráficos F, G, y A, pertenecientes a las distintas categorías cerámicas (Clara, Gris, Cocina), en los que se habían podido medir las variables descritas anteriormente. La presencia 0 ausencia de las mismas nos permitieron como en el caso anterior la catalogación de estos elementos en uno u otro Grupo Tipológico.

Sobre los bordes, (20I elementos) y por lo que respecta a las categorías cerámicas, mientras que el Grupo Tipológico I, englobaba mayoritariamente a cerámicas claras, un 74. I5\% del total del mismo, en el Grupo Tipológico II, las grises representaban un 80.82\%, al igual que el Grupo Tipológico III, también mayoritariamente Gris. Por el contrario el Grupo IV incluía cerámicas de cocina en su mayor parte. El resultado era pues interesante tanto el porcentaje de representación de estas categorías como el porcentaje de elementos en uno u otro Grupo, puesto que el resultado total constituiría, por así decirlo, la Vajilla del asentamiento. A este respecto hemos de aducir, que la abundante presencia de algunos elementos, que forman el Grupo Tipológico II, frente a los otros, nos llevaba a pensar que estabamos ante algo más que la vajilla de uso de los pobladores del lugar. Esto unido a la presencia de hornos en este asentamiento destinados a la elaboración de cerámicas, venía a confirmarnos que se estaban fabricando en este asentamiento un determinado Grupo de cerámicas (Grupo Tipológico II), que como se pretende mostrar en la Fig. 13 parecen concretarse en determinados Tipos dentro del mismo.

\footnotetext{
5 RUIZ, A; MOLINOS, M. (1989): "Fronteras: Un caso del siglo VI a.n.e. Arqueología espacial I3. Teruel, I2 I - 35

6 MOLINOS, M; RISQUEZ, C; SERRANO, J.L; MONTILLA, S.(1995): Un problema de fronteras en la periféria de Tartessos: Las Calañas de Marmolejo. Universidad de Jaén.
} 
Este primer nivel tipológico, mostraba a los dos primeros Grupos, como los más representados dentro del conjunto cerámico que se analizaba, si bien como se puede observar en las Fig. 12 y 13 presentan marcadas diferencias en el número de elementos que pertenecen a una u otra categoría. Este punto resulta muy interesante $y$ a tener en cuenta en cuanto a la función a la que pudieran haber estado destinados los recipientes.

En el Grupo Tipológico I (Fig. 12), la abundante presencia de cerámicas claras y la variedad de Tipos, nos hacía pensar en una multifuncionalidad para éstas, frente a la escasa presencia de las cerámicas de cocina o las grises. Respecto a su valoración total como Grupo, parecen incluirse, dada la diversidad de Tipos y Categorías cerámicas, distintas funcionalidades dentro del mismo. Tipos como el I, 2, 3, 13 y 14, parecen vincularse por el tamaño de sus elementos a una función más propia de almacenaje que otra cosa. Por otra parte, Tipos como el 12, parece tratarse más de una vajilla de uso, mayormente relacionada con labores culinarias. A Tipos como el 9 , ampliamente representado, $u$ otros como el I I, 7, 6, y 4, no podíamos adcribirle una determinada función participando seguramente de las ya mencionadas.

Lo que sí es interesante remarcar en este Grupo, es la amplia presencia de la cerámica clara, frente a la escasa representación que esta tenía en el resto de Grupos Tipológicos que se estudiaban. La abundancia de ésta, junto con la diversididad de formas nos llevaba a describirla como una cerámica que puede participar de muy distintos usos, relacionado con el carácter "contenedor" mientras que en el resto de los Grupos donde no aparece de forma abundante esta categoría, podría relacionarse con otros usos distintos.

Respecto al Grupo tipológico II, (Fig. 13), si bien la homogeneidad de los Tipos I, 2, 5 y
4, junto con la abundancia de los primeros nos hacía pensar en que se trataba de una "Producción" para el intercambio mayoritariamente, en la que algunos se utilizarían dentro de la vajilla del asentamiento, los Tipos individualizados (con presencia de I elemento) corresponderían al repertorio formal que compondría parte de la vajilla de uso, como lo denota la diversidad de formas destinadas probablemente a distintas funciones.

\section{LA CASA.}

Lo que pretendemos mostrar en este último ejemplo, es la aplicación que llevamos a cabo de la metodología propuesta, sobre contextos concretos: las casas del yacimiento ibérico del Cerro de la Plaza de Armas de Puente Tablas, y los espacios que se habían diferenciado dentro de ellas partiendo de una excavación microespacial de los mismos ${ }^{7}$.

Para ello se partía del estudio de dos casas (casa I y casa 2), donde se estudiaron los niveles correspondientes a los suelos de ocupación de la Fase VI-VII que cubre un período de ocupación continuado desde mediados del s. $V$ a fines del s. IV a.C. Ambas casas tienen una estructura modular semejante, con un gran patio semicubierto con acceso desde el exterior, con una compartimentación al fondo del patio, las medidas oscilan en torno a los 14 mts. de fondo y el ancho varia en relación a un segundo cuerpo creado en el lateral de la casa 2, que alcanza los $9 \mathrm{mts}$. de ancho frente a los 6 mts. que tiene la casa I, constantándose además como elemento diferenciador, la presencia de un segundo cuerpo (a partir de unas escaleras y hoyos de poste que refuerzan la tabicación interior) en la casa mayor.

En la Fig. 14, observamos el diagrama de densidad realizado sobre los análisis Factorial/Cluster, de las casas I y 2 , en la que aparecen la dispersión de tipos establecidos, sobre ella se han marcado las densidades (cada curva equivale a 5 elementos).

\footnotetext{
7 RISQUEZ, C; HORNOS, F.; RUIZ, A.; MOLINOS, M. (199I): "Aplicación del análisis multivariante: una propuesta de tipología contextualizada". Complutum I. Madrid, pp.83-98
} 
En la Fig. I5, se comparan los diagramas de densidad de las casas 1 y 2, partiendo de la dispersión de tipos que se marcaba en la figura anterior.

De estos gráficos observabamos dos hechos inmediatos. Primero que la casa $n^{\circ} \mathrm{I}$ más pequeña, reducía sensiblemente su área de dispersión en el gráfico respecto a la casa 2 (Fig I5). Este hecho que supone en la práctica la definición de una vajilla más limitada para la primera casa que para la segunda está en relación directa con el tamaño, $(14 \times 5)$ en la primera frente $(14 \times 9)$ en la segunda. En segundo lugar y por la comparación de ambos casos, las máximas densidades en la primera casa son distintas a la segunda por la mayor presencia de fragmentos de recipientes cerrados y la disminución de los recipientes abiertos, que adquieren altas densidades en la casa $n^{\circ} 2$. En su momento este hecho permitía que formulásemos una pregunta ¿Podría valorarse este hecho indicativo de la pobreza del conjunto material, como un efecto de la posición social de los dos grupos que habitaban en cada casa?. Señalábamos aquí la presencia en la casa 2 crátera ática de figuras rojas.

Por último en la Fig. 16, se puede comparar la dispersión de esos tipos en distintos espacios de la casa 2, correspondiendo los espacios 8-3 y 9 a habitaciones, mientras que el tercero se identifica como patio.

Una rápida lectura sobre esas habitaciones de la casa 2 nos hacía valorar la diferencia entre los espacios 8-3 y 9, que conteniendo preferentemente recipientes abiertos, siguen canones diferentes en las tendencias de las formas de borde que cada una contiene (Subt. 14 para el espacio 8-3 y Subt. 16 y 17 para el espacio 9 según los análisis realizados en su momento) lo que expresado en tendencias morfométricas venía a mostrar un tipo de paredes más verticales en el espacio 9 que en el 8-3. El espacio 8-3 por su parte estaba reproduciendo a pequeña escala un sistema de densidades que recordaba el existente en el patio, con lo que teníamos un modelo funcional semejante en el área abierta y cubierta de la casa. Aunque parece evidente que los grandes recipientes contenedores de líquidos (ánforas) se disponen en el patio y no en la zona interior, ya que es allí donde han sido localizados en el proceso de excavación, resultaba interesante valorar que las grandes concentraciones de la casa en peso y número total de fragmentos se localizan precisamente en el patio y en el espacio 8-3 RUIZ y MOLINOS 1989.

El patio, por su parte, permitía debido a su gran dimensión valorar un sistema multifuncional en razón a la dispersión espacial de los fragmentos que advertía sobre un área de producción para el consumo-almacenaje y otra propiamente de consumo. Todo ello nos llevaba a pensar en un circuito de actividad estival en el área abierta y otro más distanciado que articularía exterior-interior para la etapa de invierno. Este proceso podría haberse producido sin distinción (invierno-verano) en la casa I ya que los recipientes cerrados se localizaban preferentemente en el patio en tanto que los abiertos parecen concentrarse en el interior.

Con todo esto y a partir de estos ejemplos que hemos presentado esperamos haber dado respuesta a la pregunta que nos formulábamos y que sirvió de base para desarrollar lo que hasta aquí hemos expuesto. Con todo ello, hemos de decir, que la metodología que hemos presentado, no está ni mucho menos cerrada, sino que ha sido un paso más en el camino de la cuantificación, pero que EL CAPÍTULO QUEDA ABIERTO.

\section{B I B L I O G R A Fí A}

ALDENDEFER,M.S. (1987): "Quantitative Research in Archaeology: Progress and prospects". Sage, Newsbury Park.

ARROYO-BISHOP, D. (199I): "The Archeo-data system - towards a Europeam archaeological document". Computer applications and quantitatives methods in archeaology, 1990. B.A.R. International Series 565. Oxford, pp. 61-70.

BARCELÓ, J.A. (1989): "Introducción al razonamiento estadístico aplicado a la Arqueología: un análisis de las estelas antropomorfas de la Peninsula Iberica". Trabajos de Prehistoria, 45. Madrid. 
BATISTA, I.M. (1984): "Componentes principales y Análisis Factorial (Exploratorio y confirmatorio)". En J.J. Sánchez Carrión (Ed.). Introducción a las Técnicas de Análisis Multivariable aplicadas a las Ciencias Sociales. Centro de Investigaciones Sociológicas. Madrid, pp. 23-74.

BINFORD, L. (1972): An Archaeological Perspective. Accademic Press. N. Y.

BISQUERRA ALZINA, R. (1989): Introduccion conceptual al analisis multivariable. Vol.I-II. P.P.U. Barcelona.

BMDP. STATISTICAL SOFTWARE (1983): Printing with Additions. Ed. W.J. Dixon. University of California Press. Berkeley.

CASTRO, M.,LÓPEZ, J.; ZAFRA, N.; CRESPO, J.; CHOCLÁN, C. (1987): "Prospección con sondeo estratigráfico en el yacimiento de Atalayuelas, Fuerte del Rey, Jaén." Anuario Arqueológico de Andalucía, II. Sevilla. pp. 207-215

CLARKE, D.L.(1984): Arqueología Analítica. Bellaterra. Barcelona

CONTRERAS, F.(1984): "Clasificación y tipología en Arqueología.El camino hacia la cuantificación". Cuadernos de Prehistoria Universidad de Granada. No 9. Granada. pp.327-385

CONTRERAS,F.(1986): "Aplicación de métodos estadísticos y analíticos aplicados a los complejos cerámicos de la Cuesta del Negro.Purullena-Granada". Tesis Doctorales de la Univ.Granada.

CONTRERAS, F. (1988): "El papel actual de la informática en la Arqueología". Estudis Balearics, N²4, pp. 289298.

CONTRERAS, F. (1994): "Una aproximación a los estudios tipológicos. La cerámica." Actas del II Congreso de Historia de Andalucía. Cordoba.pp 37-46

CONTRERAS, F; CAPEL, J; ESQUIVEL, J.A.; MOLINA, F; de la TORRE, F. (1988): "Los ajuares cerámicos de la necrópolis argárica de la Cuesta del Negro (Purullena, Granada). Avance al estudio analítico y estadístico." Cuadernos de Prehistoria Universidad de Granada. No $12-$ 13. Granada. pp.|35-155

CONTRERAS, F., MOLINA, F. y ESQUIVEL, J.A. (I99|) Propuesta de una metodología para el estudio tipológico de complejos arqueológicos mediante análisis multivariante. Aplicaciones Informáticas en Arqueología, Complutum I, Madrid, pp. 65-82.

CRESPO, J.; CASTRO, M.; LÓPEZ ,.; CHOCLÁN,C. (1986): "Prospección arqueológica con sondeo en la finca Gil de Olid, Puente del Obispo, Baeza (Jaén)". Anuario Arqueológico de Andalucía, II. Sevilla.

CUADRAS, C.M. (198|): Métodos de Análisis Multivariante. Ed. Universitaria de Barcelona. Barcelona.

CHAPA,T. (1984): "Aspectos metodológicos de la tipología arqueológica:un ejemplo referido a las fibulas dela Téne". I Jomadas de Investigación Prehistórica, Soria 198I. Madrid
DÍAZ-ANDREU, M. y MONTERO, I. (|99|): "Sistemas de representación gráfica por ordenador: mapas de densidades de materiales arqueológicos." Aplicaciones Informáticas en Arqueología. Complutum I. Madrid, pp.299-304.

DJINDJIAN, F. (1984-85): Informatique et mathematiques appliquees en archeologie: une introduction. Antiquites Nationals, 16/17.

DORAN, J.E. y HODSON, F.R. (1975): Mathematics and Computers in Archaeology. Edimburgh University Press.

ESQUIVEL, J.A. y CONTRERAS, F. (I984): "Una experiencia arqueológica con microordenadores.Análisis de componenetes Principales y Clusterización: Distancias Euclídeas de Mahalanobis". Congreso Nacional XIV de Estadística, Investigación Operativa e Informática. Granada.

ESQUIVEL, I.A., CONTRERAS, F., MOLINA, F. y CAPEL, J. (1991): "Una aplicación de la teoría de la información al análisis de datos definidos mediante variables cualitativas multiestado: medidas de similaridad y análisis cluster". Aplicaciones Informáticas en Arqueología. Complutum I. Madrid, pp. 53-64.

ESTÉVEZ, J. y LULL, V. (1984): "Aplicación de la biometría elemental al análisis cerámico". I Jornadas de Metodología de Investigación Prehistórica. Soria 1981. Madrid.

FERNÁNDEZ, V.M. (1985): "La seriación automática en arqueología: introducción histórica y aplicaciones". Trabajos de Prehistoria. 42 Madrid.

FERNÁNDEZ, V.M. y FERNÁNDEZ, G. (|99|): "El sistema Tiesto: una propuesta de análisis de los fragmentos cerámicos en excavaciones arqueológicas". Aplicaciones Informáticas en Arqueología. Complutum I. Madrid. pp. 231-242.

GRACIA, Ma et al. (1984): "Análisis de la fragmentación cerámica para el establecimiento de tablas de formas y su evolución cronológica". I Jornadas de Metodología de Investigación Prehistórica. Soria 1981. Madrid.

GUTIÉRREZ, L.M. (1994): El oppidum de Giribaile: un análisis de microespacio en superficie. Memoria de Licenciatura. Universidad de Granada. Inédita.

HODSON, F. (1970): Cluster analysis in Archaeology: some new developments and applications. World Archaeology, 1,3.

HODSON, F. (1980): "Cultures as types? some elements of classification theory". Institute of Archaeology Bulletin, 17.

HORNOS MATA, F. (1984): La necrópolis ibérica de la Finca de Gil de Olid (Puente del Obispo, Baeza). Memoria de Licenciatura (inédita). Universidad de Granada.

KENDALLD.G. (1975): Analysis Multivariant. Griffin \& Co. Bristol. LÓPEZ ROZAS, J.(1984): El horizonte protoibérico del Cerro de la Coronilla, Cazalilla, Jaén. Memoria de Licenciatura (inédita). Universidad de Granada. 
LORRIO, A.J. (|99|): "Clasificación automática de formas completas: un estudio comparativo de diversos métodos multivariantes". Aplicaciones informáticas en Arqueología. Complutum I. Madrid, pp. 99-I 12.

LULL, V. (1983):La cultura de El Argar ed. Akal. Barcelona.

MARTíN DE LA CRUZ, J.C. (1987): "El LLanete de los Moros, Montoro, Córdoba". Excavaciones Arqueológicas en España. 151. Madrid.

MOLINOS, M. (1987): Poblamiento ibérico en la Campiña Oriental de Jaén. Tesis Doctoral. Universidad de Granada.

MOLINOS, M.; SERRANO, J.L.; COBA, B. (I 988): "Excavaciones arqueológicas en el asentamiento de La Campiña, Marmolejo (Jaén)". Anuario Arqueológico de Andalucía, III. Sevilla

MOLINOS, M; RÍSQUEZ, C; SERRANO, J; MONTILLA, S. (1995): Un problema de fronteras en la periferia de Tartessos: Las Calañas de Marmolejo. Universidad de Jaén.

NICOLINI, G.; RUIZ, A.; ZAFRA, N. (1987): Informe sobre la Campaña de Excavación arqueológica de 1987 en los Altos del Sotillo, Castellar de Santisteban, Jaén". Anuario Arqueológico de Andalucía. II, Sevilla.
NOCETE, F.(1990): El espacio de la coerción. La Transición al Estado en las Campiñas del Alto Guadalquivir. España 3.000-I.500 a.n.e. BAR. M.S.P.A., I .

ORTON, C. (1988): Matemáticas para arqueólogos. Alianza Universidad. Madrid.

RÍSQUEZ, C. (1993): Las cerámicas de cocción reductora en el Alto Guadalquivir durante la época ibérica: Hacia una tipología contextual. Tesis Doctoral. Universidad de Granada. Microfichas.

RÍSQUEZ, C., HORNOS, F., RUIZ, A. y MOLINOS, M. (199I) Aplicación del Análisis Multivariante, una propuesata de tipología contextualizada. Aplicaciones Informáticas en Arqueología. Complutum I. Madrid, pp. 83-98.

RUIZ, A.; MOLINOS, M. (1989): "Estudio de materiales de la campaña arqueológica de 1988". Anuario Arqueológico de Andalucía. II. Sevilla.

RUIZ, A.; MOLINOS, M. (1989): "Fronteras: un caso del siglo VI a.n.e." Fronteras. Arqueología Espacial, I3. Teruel, pp. $|2|-\mid 35$.

SÁNCHEZ CARRIÓN, J.J. (1984): Introducción a la técnica de Análisis multivariable apliacada a las Ciencias Sociales. Centro de Investigaciones Sociológicas. Madrid. 


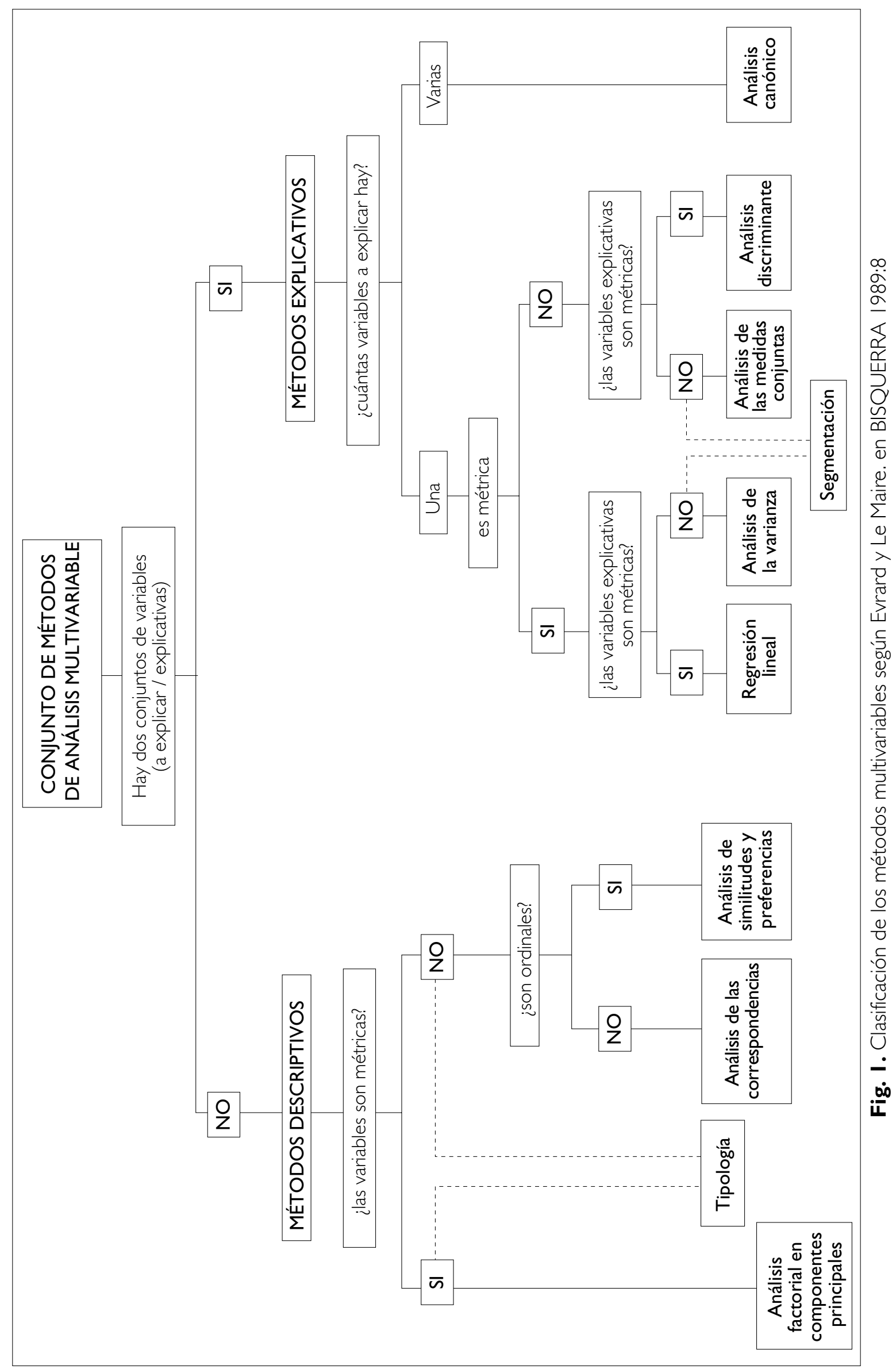




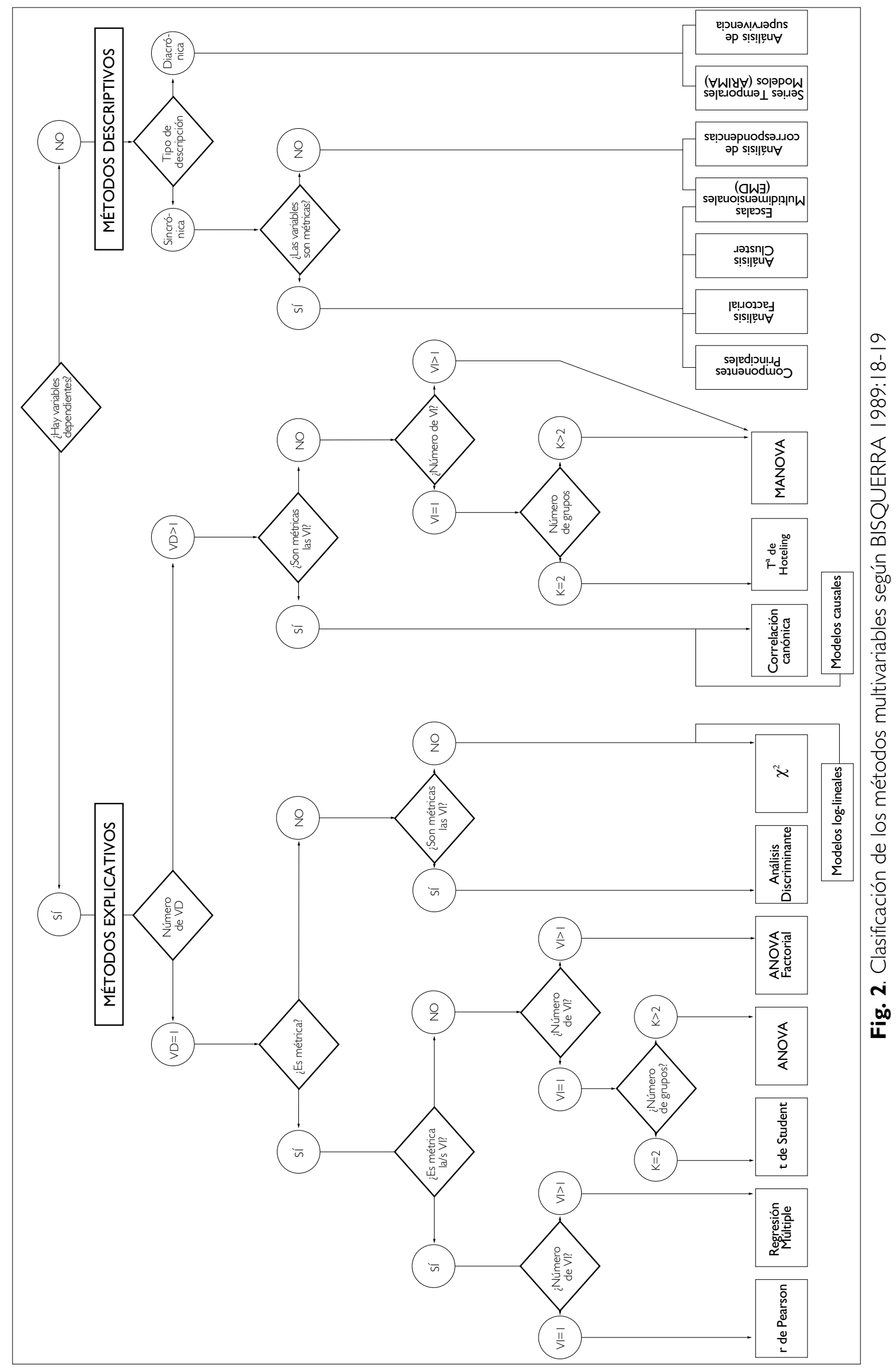




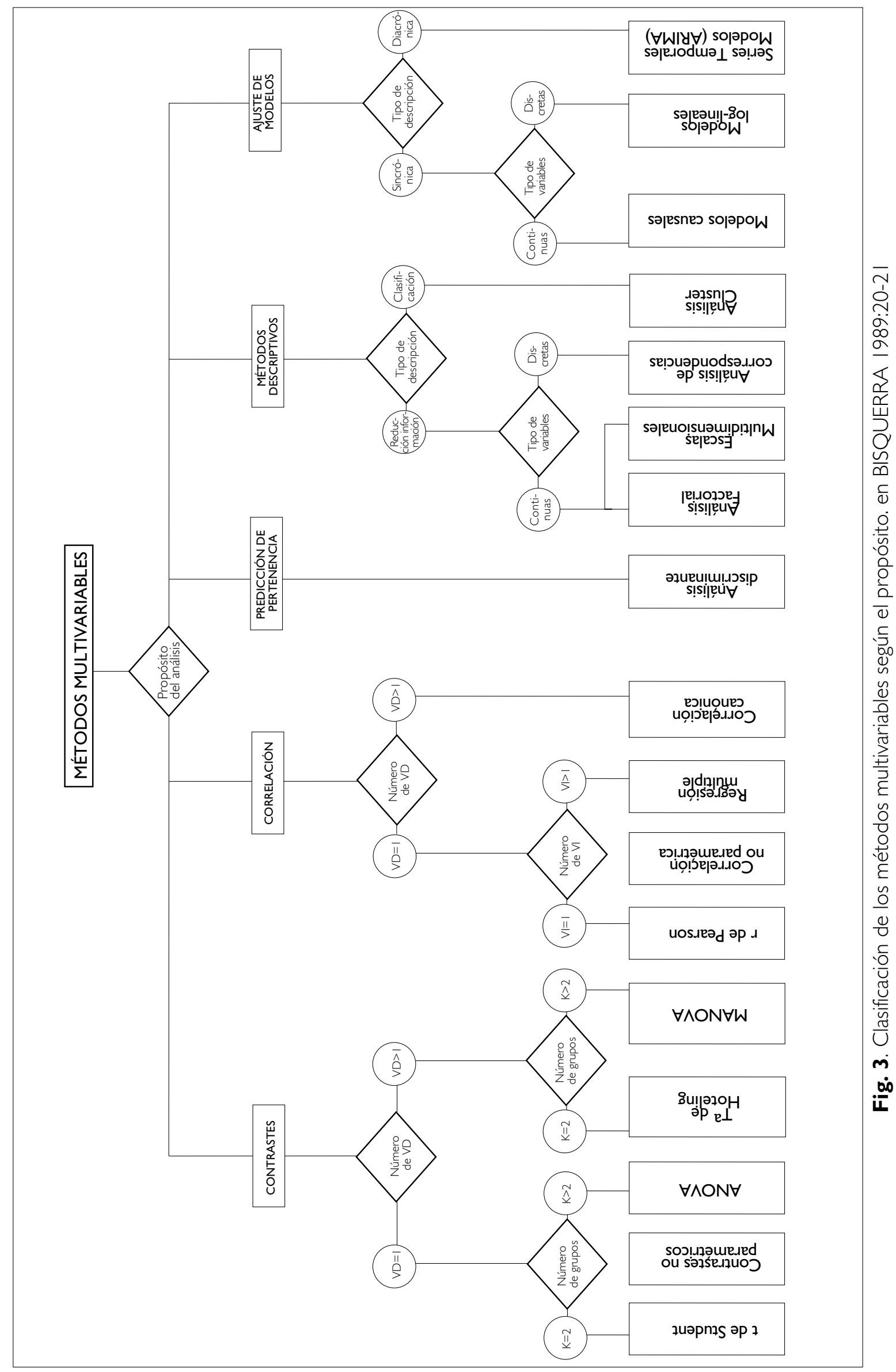




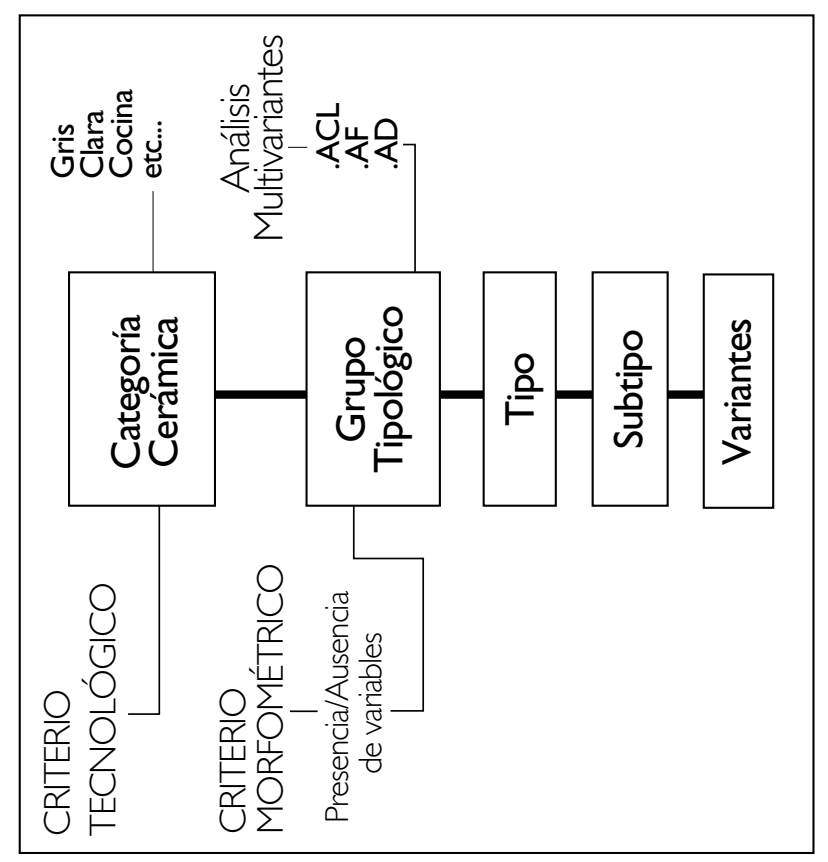

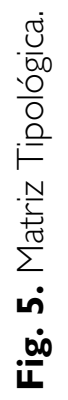

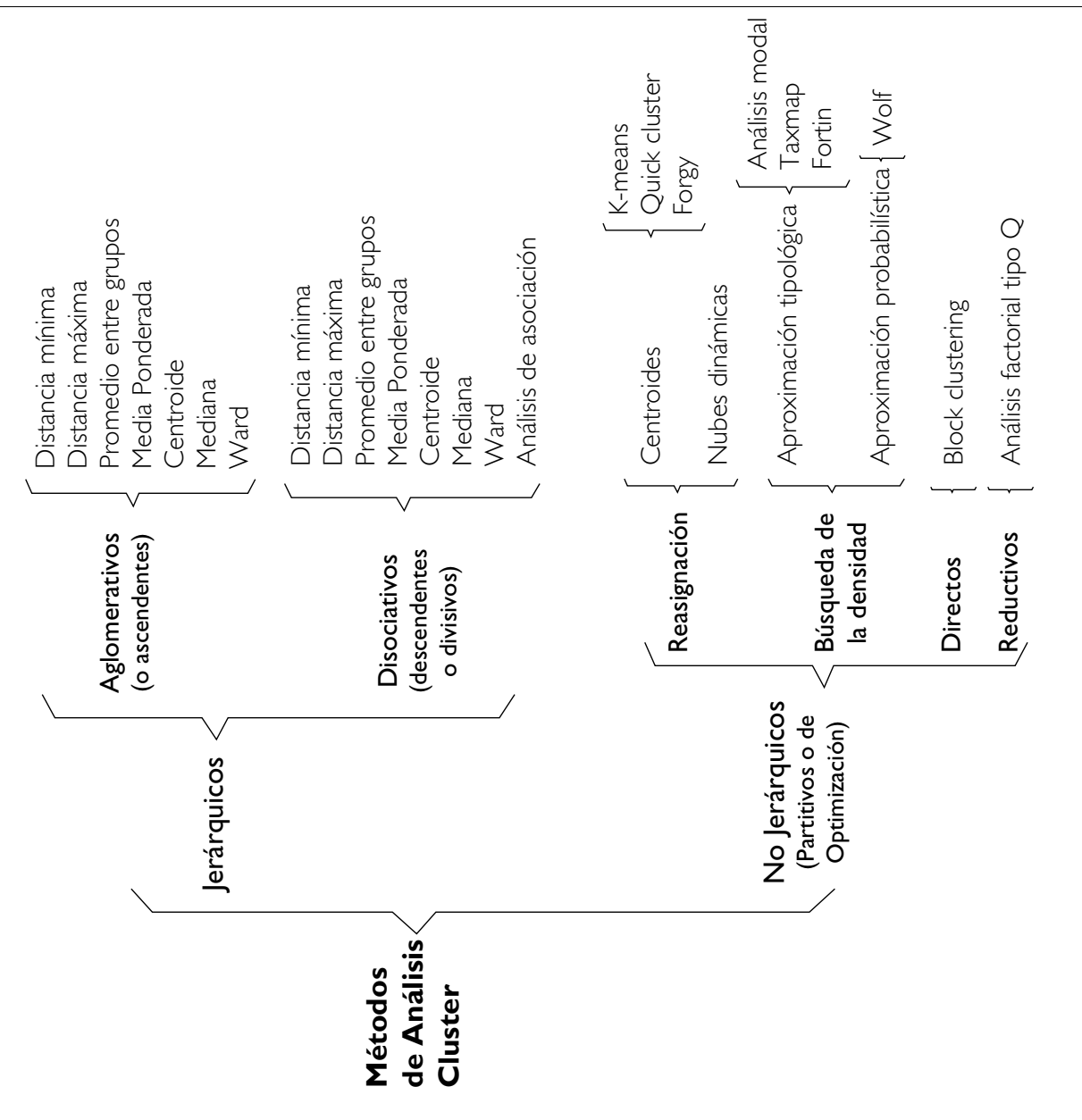

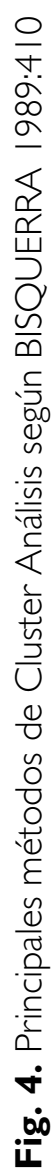




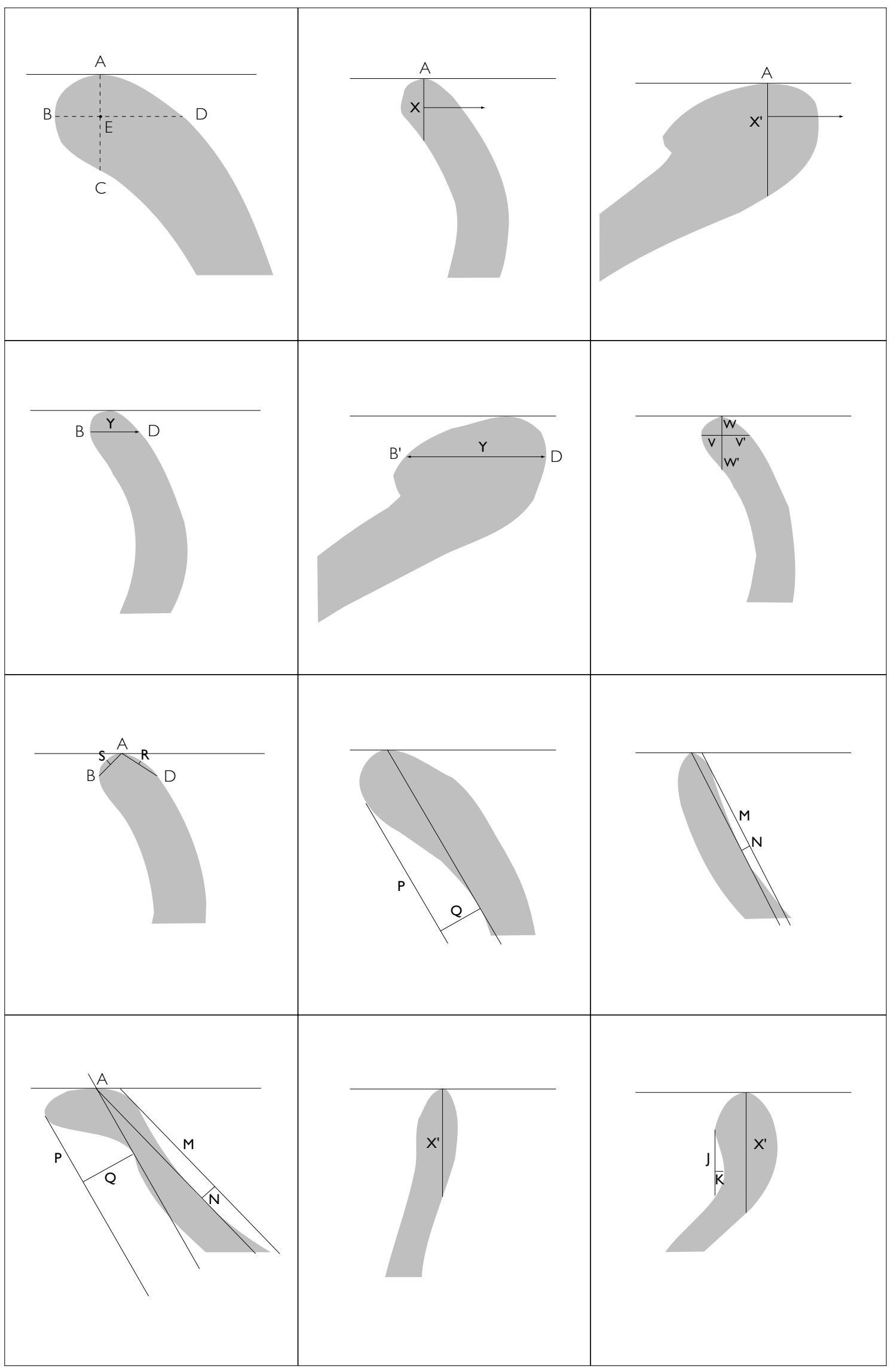

Fig. 6. Variables morfométricas utilizadas para los bordes. 


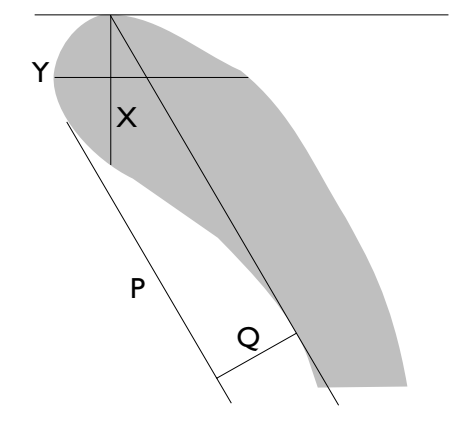

GRUPO TIPOLÓGICO I

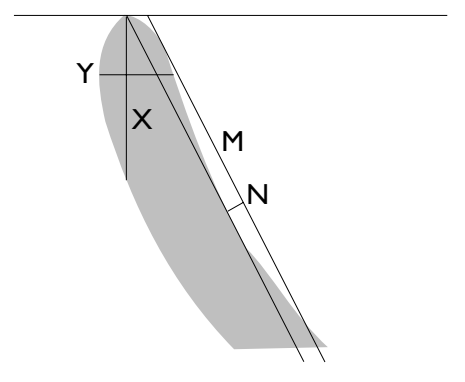

GRUPO TIPOLÓGICO II
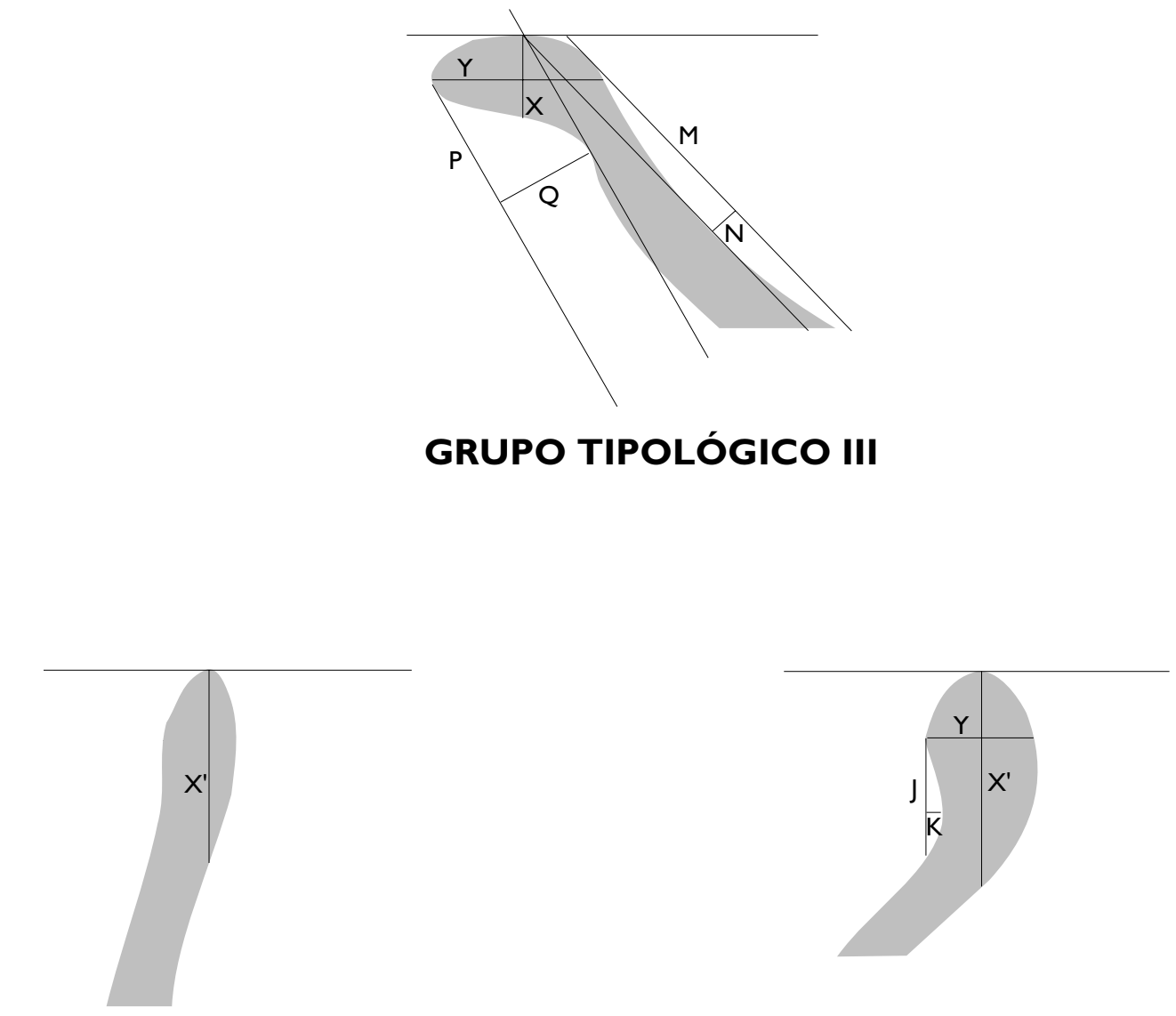

GRUPO TIPOLÓGICO IV GRUPO TIPOLÓGICO V

Fig. 7. Grupos tipológicos de bordes a partir de la presencia o ausencia de las variables propuestas. 


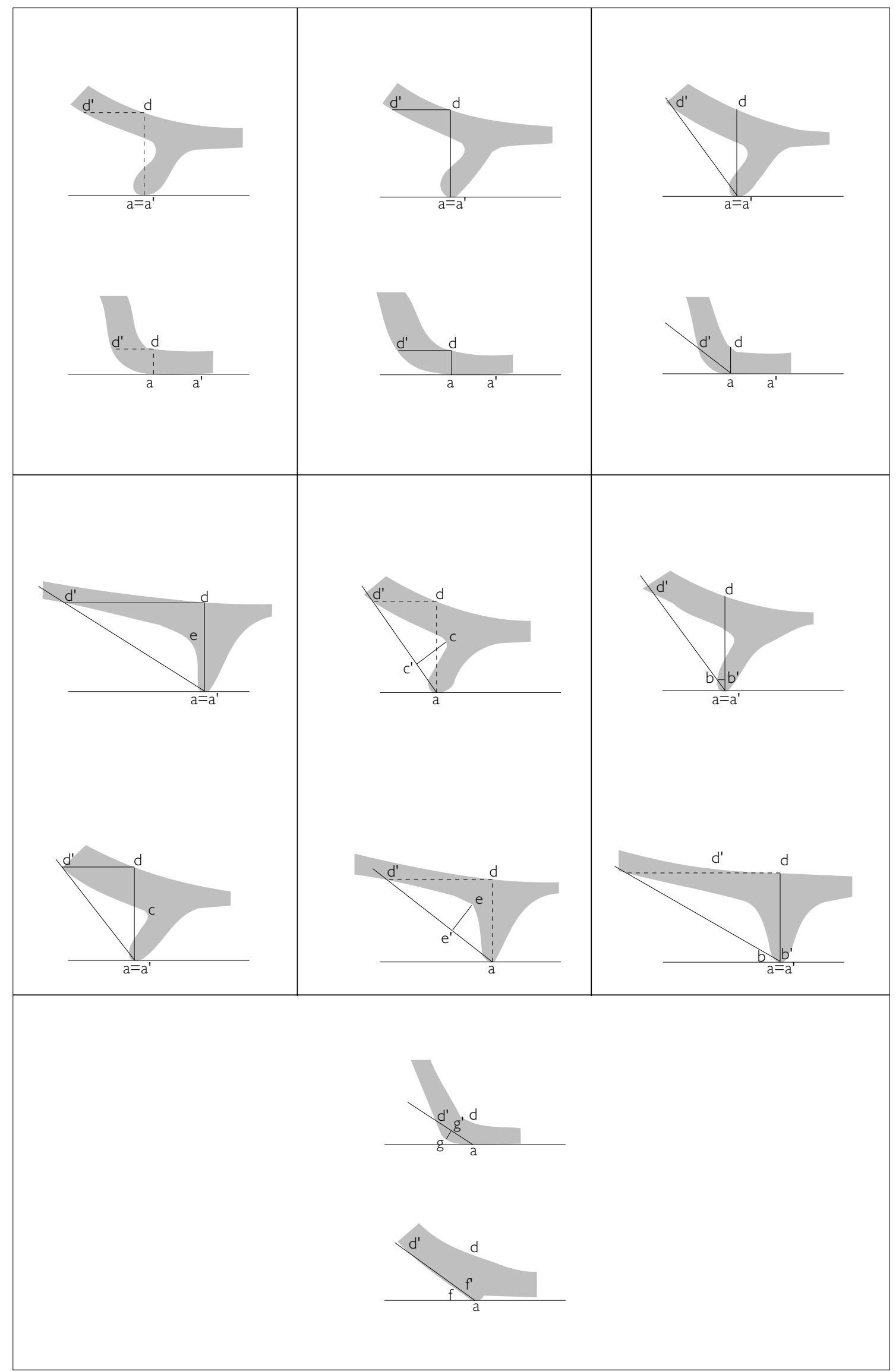

Fig. 8. Variables morfométricas utilizadas para las bases. 


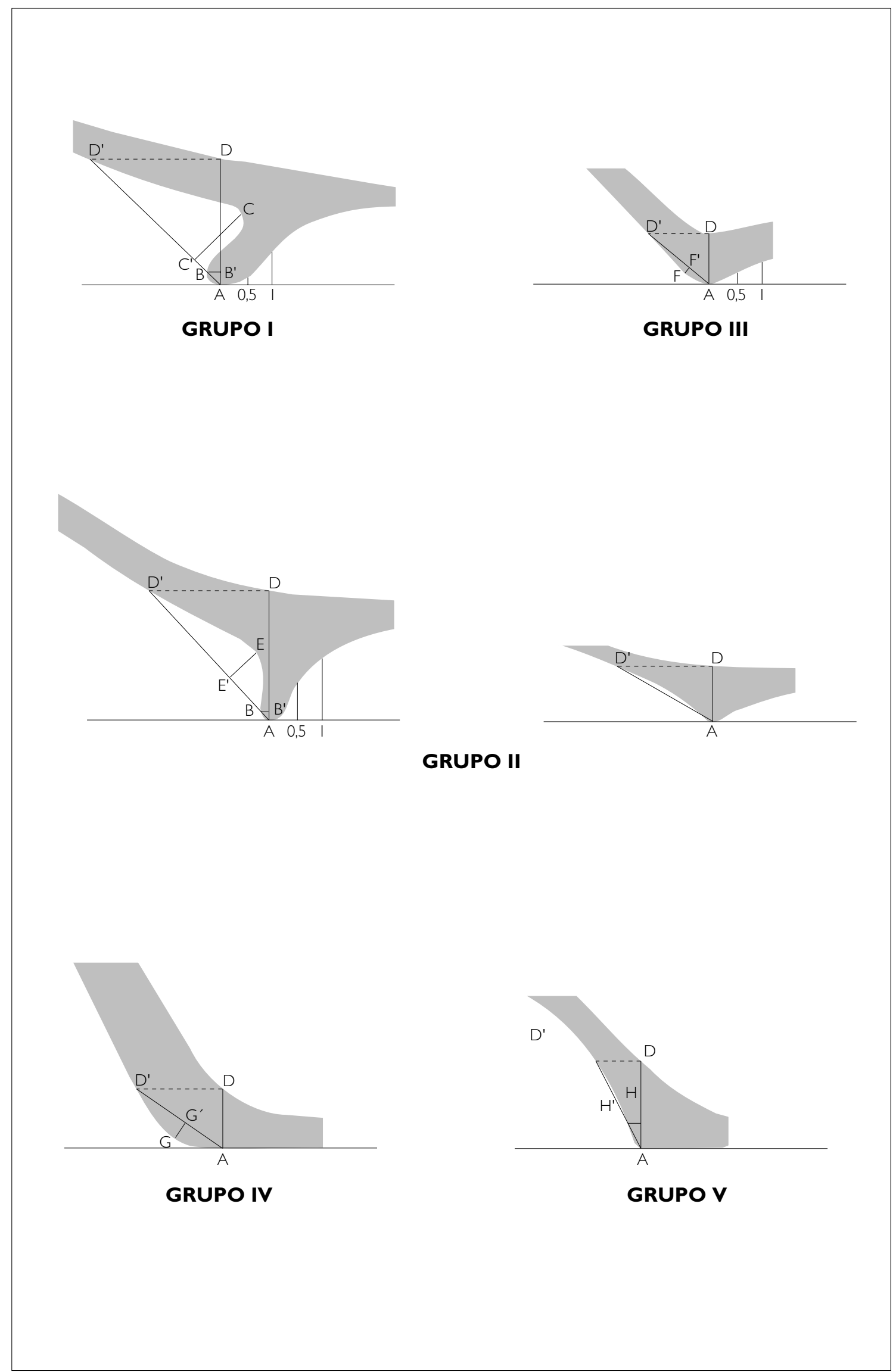

Fig. 9. Grupos tipológicos de bases a partir de la presencia o ausencia de las variables propuestas. 


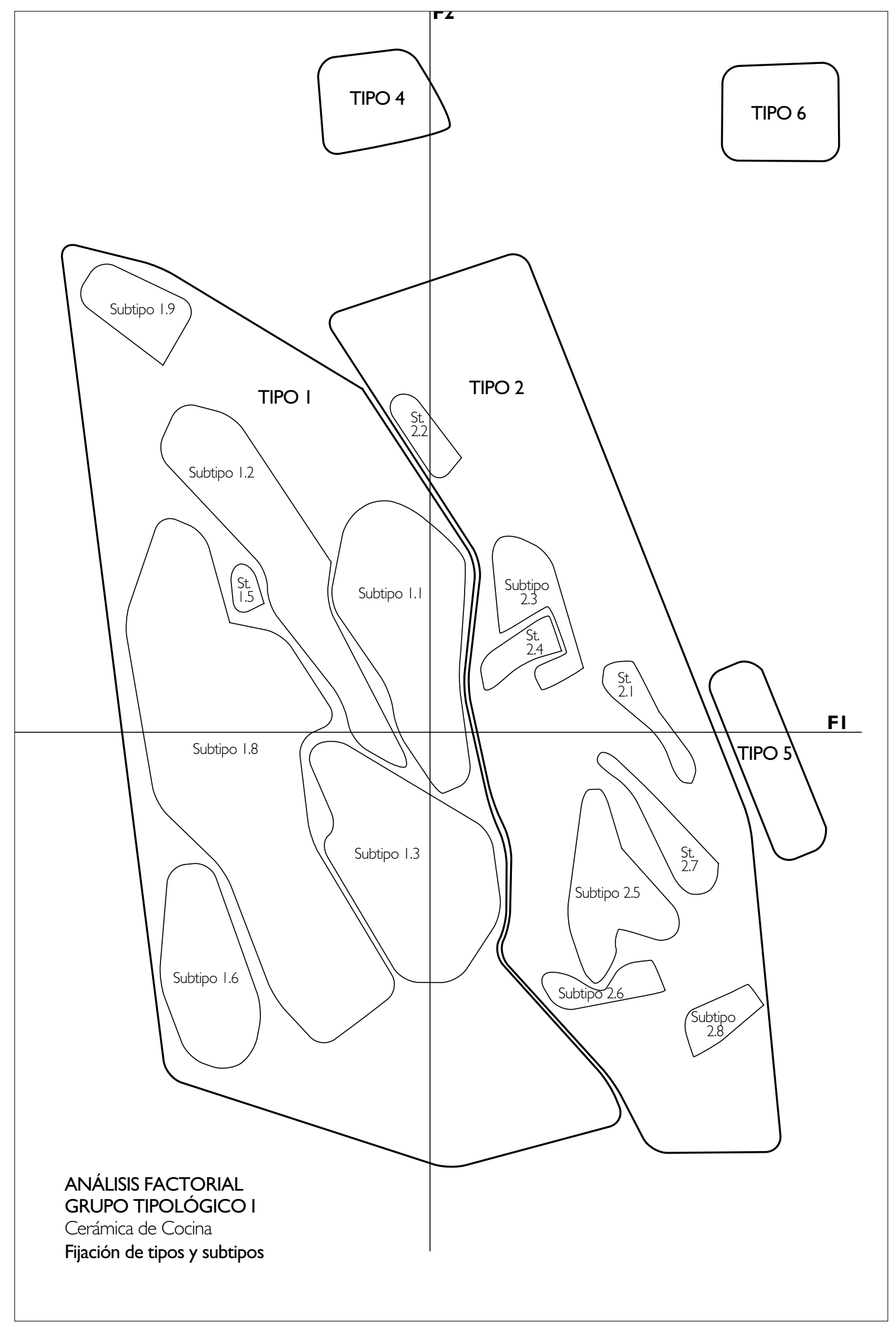

Fig. 10. Resultados obtenidos para el Grupo Tipológico I de cerámicas de cocina (bordes). en Rísquez 1993 


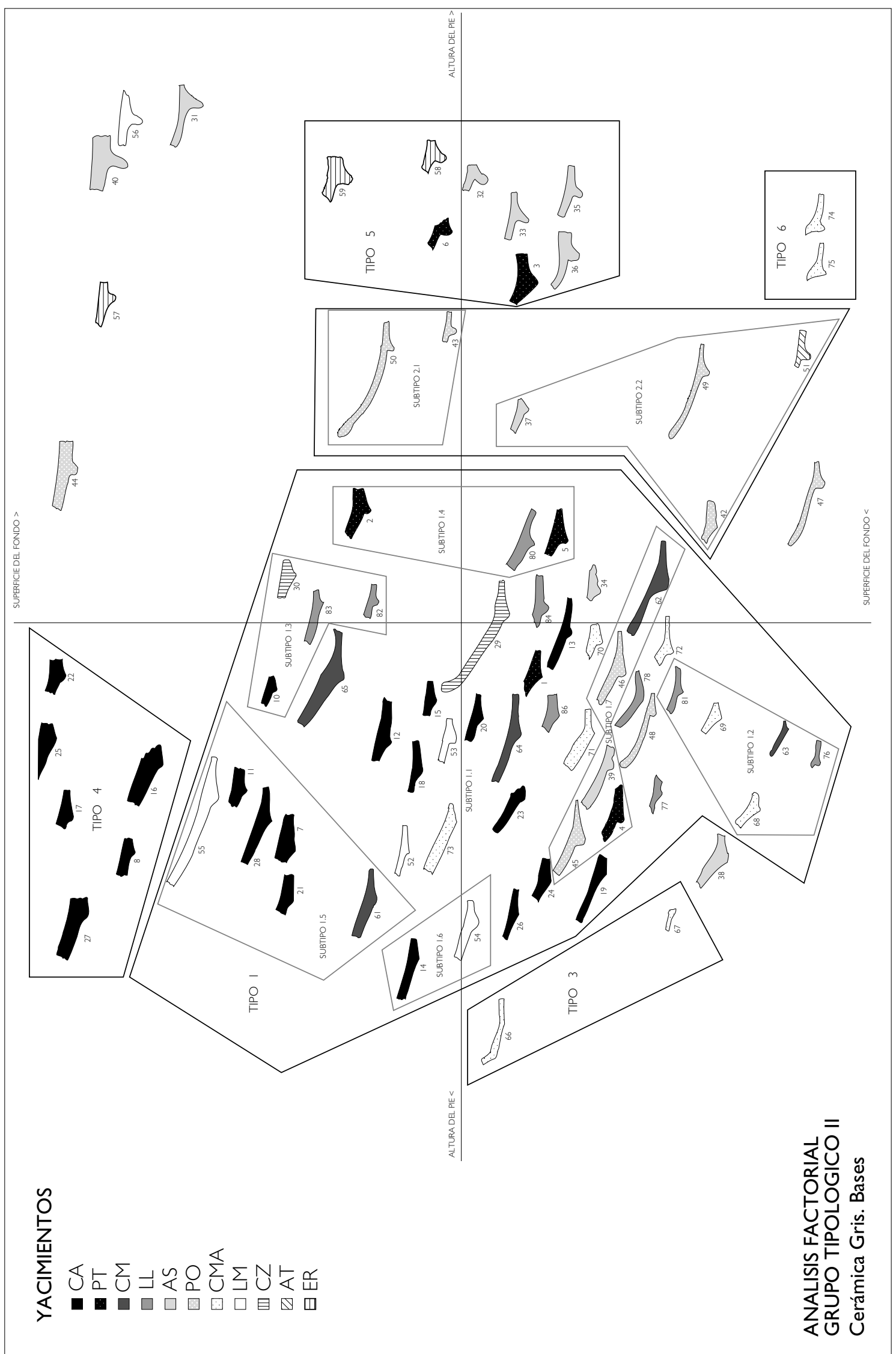

Fig. I I. Resultados obtenidos para el Grupo Tipológico II de cerámicas grises (bases). en Rísquez 1993 


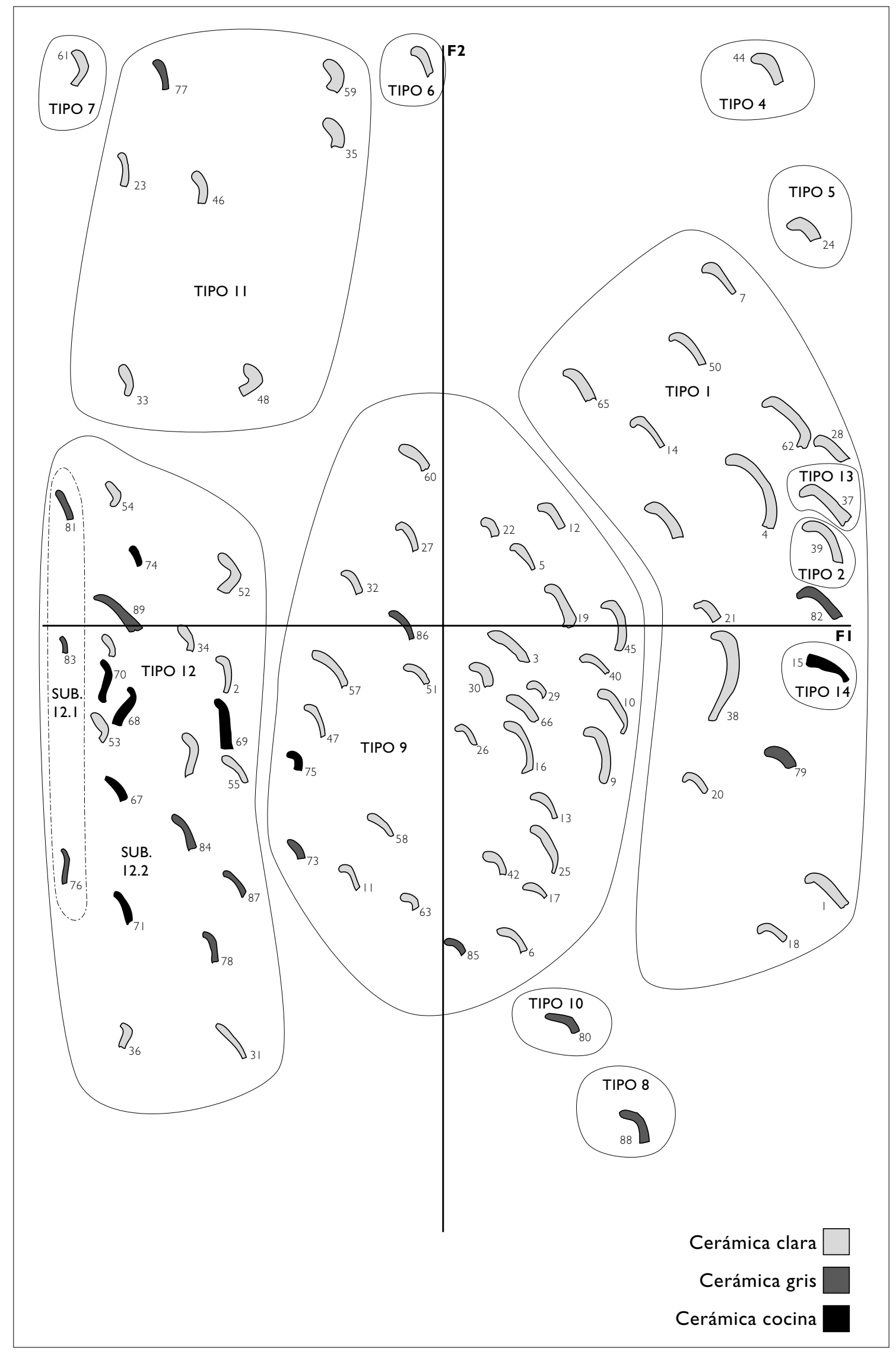

Fig. I 2. Tipos establecidos para el Grupo Tipológico I (bordes) en el Yacimiento de Las Calañas. Marmolejo. en Molinos et al. 1995 


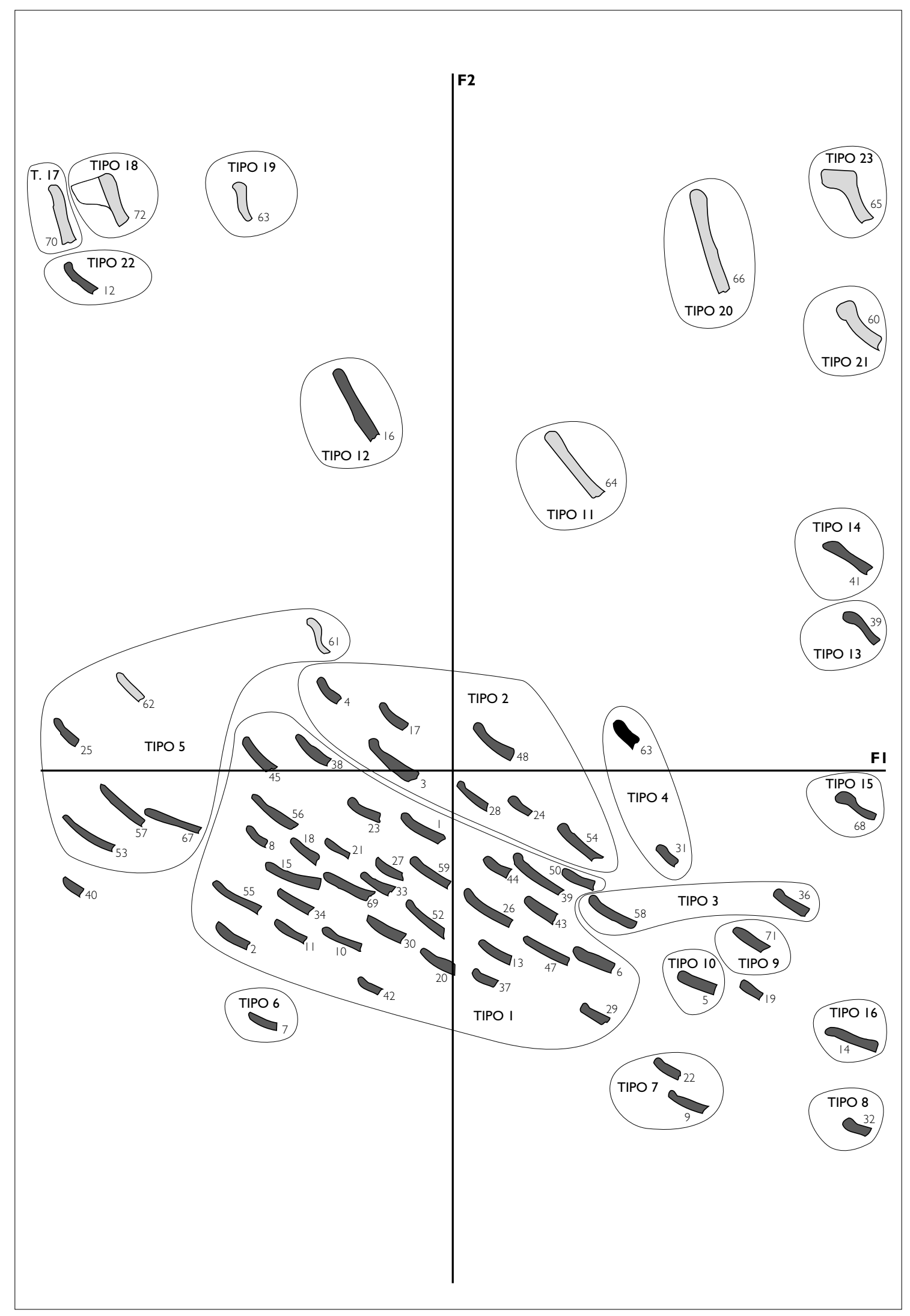

Fig. 13. Tipos establecidos para el Grupo Tipológico II (bordes) en el Yacimiento de Las Calañas. Marmolejo. en Molinos et al. 1995 


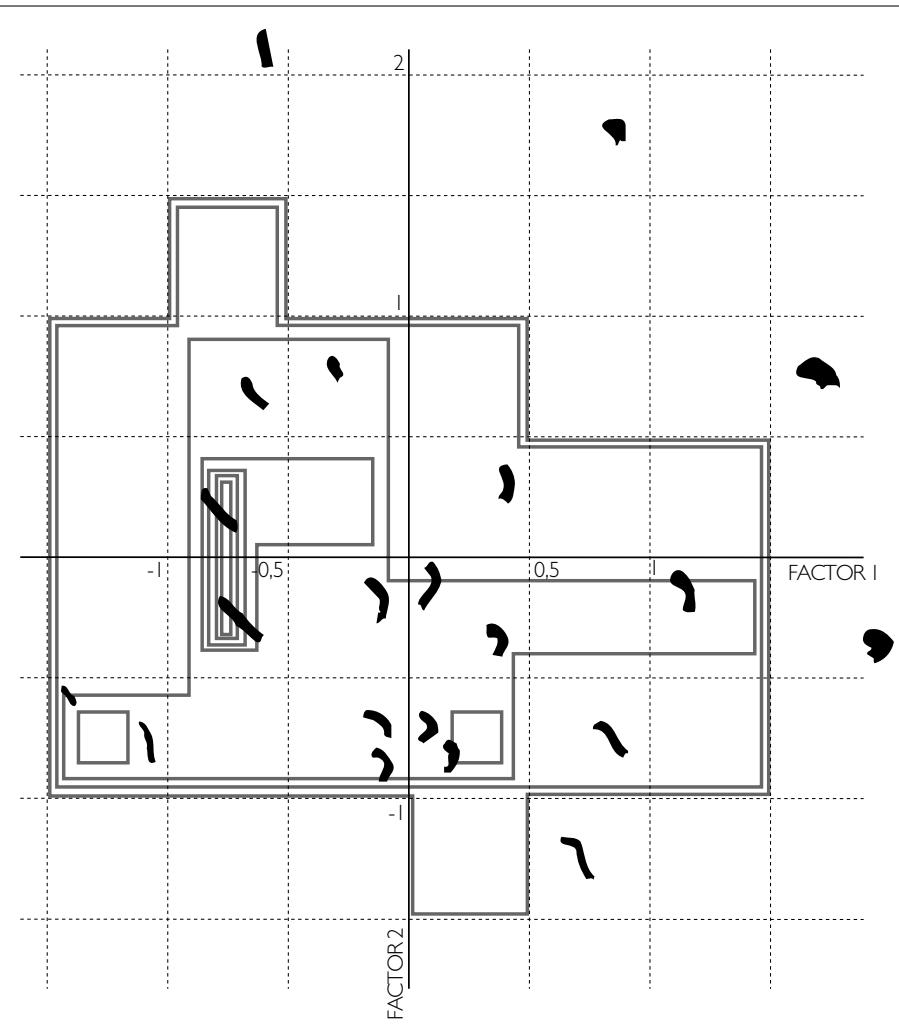

Fig. 14. Diagrama de densidad para las casa I y 2 del Yacimiento del Cerro de la Plaza de Armas de Puente Tablas. Jaén. en Rísquez et al. 1991
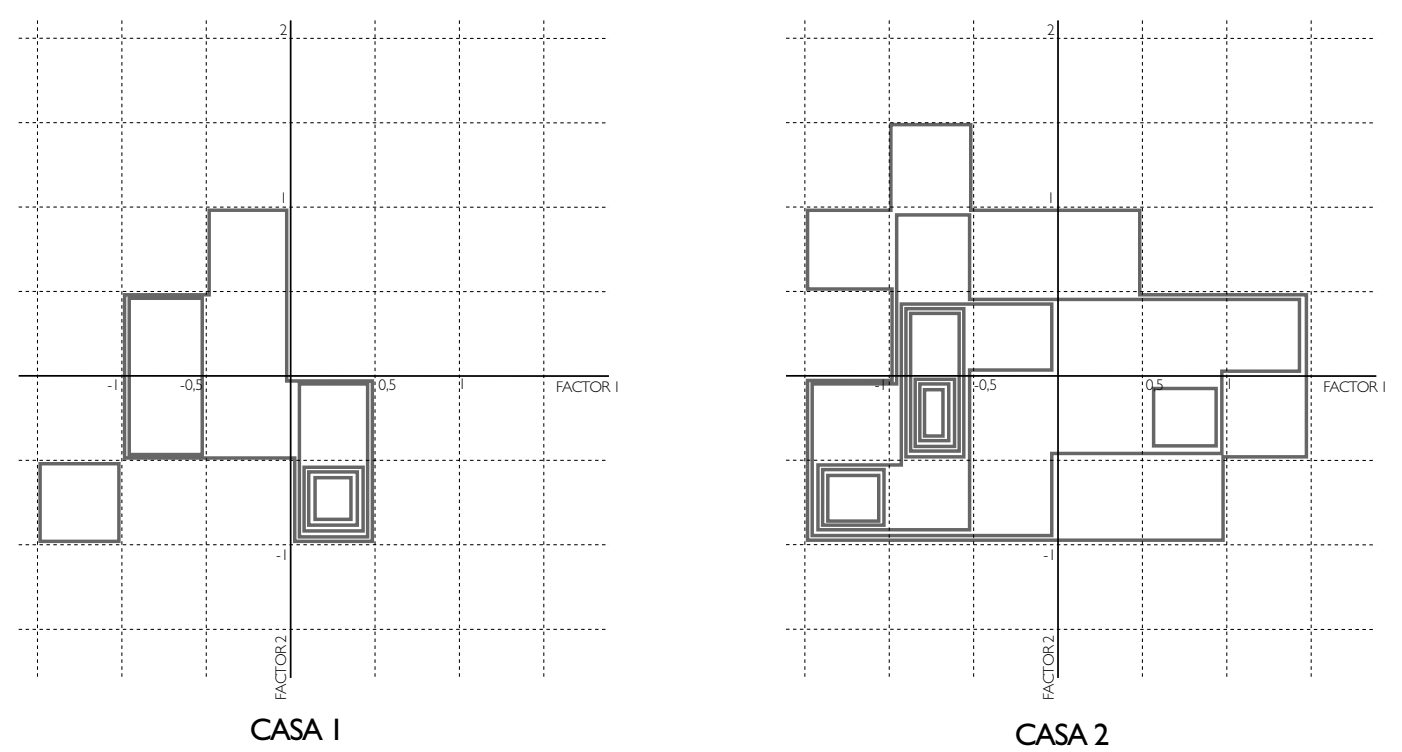

Fig. I5. Comparación a partir de diagramas de densidad de las casas l y 2 según el repertorio cerámico que presentan. en Rísquez et al. 1991 


\section{Espacio 8-3}

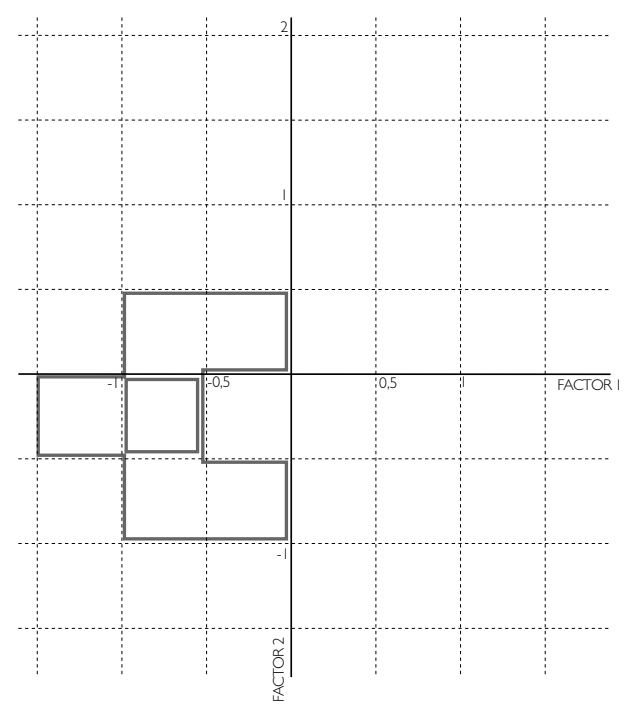

Espacio 9

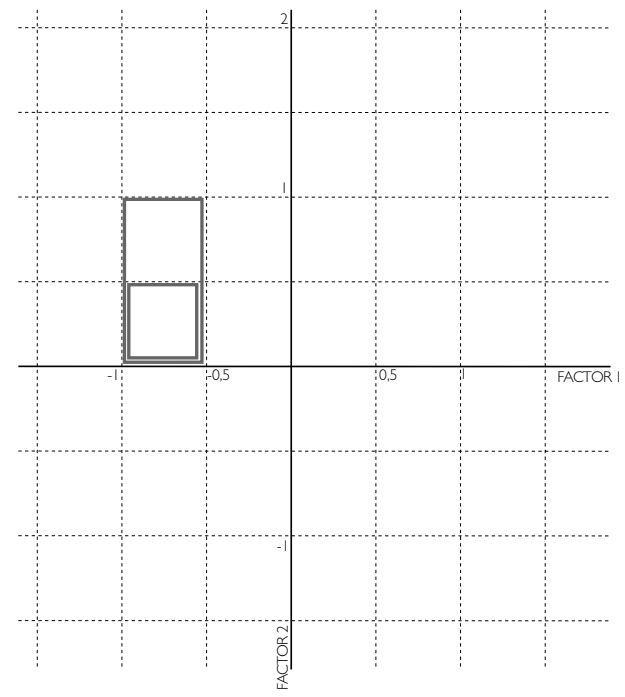

Patio

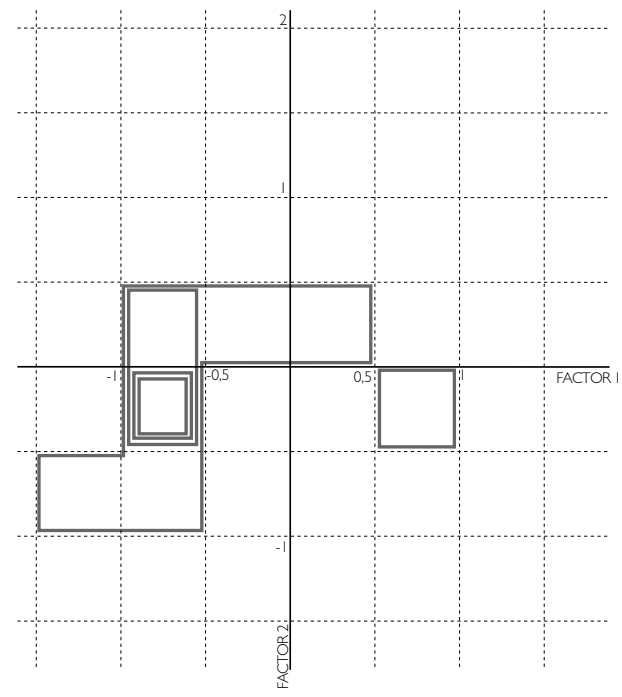

Fig. 16. Comparación de los espacios 8-3, 9 y patio de la casa 2, donde se puede observar la variación que se produce entre ellos. en Rísquez et al. |99| 